\title{
CHEMICAL AND PHOTOMETRIC EVOLUTION OF EXTENDED ULTRAVIOLET DISKS: OPTICAL SPECTROSCOPY OF M83 (NGC 5236) AND NGC $4625^{1}$
}

\author{
Armando Gil de Paz, ${ }^{2,3}$ Barry F. Madore, ${ }^{2,4}$ Samuel Boissier, ${ }^{2,5}$ David Thilker, ${ }^{6}$ Luciana Bianchi, ${ }^{6}$ \\ Carmen Sánchez Contreras,,${ }^{7,8}$ Tom A. Barlow, ${ }^{8}$ Tim Conrow,${ }^{8}$ Karl Forster, ${ }^{8}$ Peter G. Friedman, ${ }^{8}$ \\ D. Christopher Martin, ${ }^{8}$ Patrick Morrissey, ${ }^{8}$ Susan G. Neff, ${ }^{9}$ R. Michael Rich, ${ }^{10}$ David Schiminovich, ${ }^{8}$ \\ Mark Seibert, ${ }^{8}$ Todd Small, ${ }^{8}$ José Donas, ${ }^{4}$ Timothy M. Heckman, ${ }^{11}$ Young-Wook Lee, ${ }^{12}$ \\ Bruno Milliard, ${ }^{4}$ Alex S. Szalay, ${ }^{11}$ Ted K. Wyder, ${ }^{8}$ and Sukyoung Yi ${ }^{12}$ \\ Received 2006 July 28; accepted 2007 February 11
}

\begin{abstract}
We present the results from the analysis of optical spectra of $31 \mathrm{H} \alpha$-selected regions in the extended UV (XUV) disks of M83 (NGC 5236) and NGC 4625 recently discovered by GALEX. The spectra were obtained using IMACS at the Las Campanas Observatory $6.5 \mathrm{~m}$ Magellan I telescope and COSMIC at the Palomar 200 inch (5 m) telescope, respectively, for M83 and NGC 4625. The line ratios measured indicate nebular oxygen abundances (derived from the R23 parameter) of the order of $Z_{\odot} / 5-Z_{\odot} / 10$. For most emission-line regions analyzed the line fluxes and ratios measured are best reproduced by models of photoionization by single stars with masses in the range $20-40 M_{\odot}$ and oxygen abundances comparable to those derived from the R23 parameter. We find indications for a relatively high $\mathrm{N} / \mathrm{O}$ abundance ratio in the XUV disk of M83. Although the metallicities derived imply that these are not the first stars formed in the XUV disks, such a level of enrichment could be reached in young spiral disks only 1 Gyr after these first stars would have formed. The amount of gas in the XUV disks allows maintaining the current level of star formation for at least a few Gyr.
\end{abstract}

Subject headings: galaxies: abundances — galaxies: evolution $-\mathrm{H}$ II regions - techniques: spectroscopic ultraviolet: galaxies

Online material: color figures

\section{INTRODUCTION}

Studying the outer edges of spiral galaxies provides a unique tool for understanding the formation and evolution of galactic disks. According to the inside-out scenario of disk formation, an increase in the gas infall timescale with the galactocentric radius results in the delayed formation of the stars in the outer parts of the disk compared with its inner regions (Larson 1976; Matteucci \& Francois 1989; Hou et al. 2000; Prantzos \& Boissier 2000).

\footnotetext{
${ }^{1}$ Based in part on observations made at the Magellan I (Baade) telescope, which is operated by the Carnegie Institution of Washington.

2 The Observatories, Carnegie Institution of Washington, Pasadena, CA 91101; agpaz@ociw.edu.

3 Departamento de Astrofísica, Universidad Complutense de Madrid, Madrid 28040, Spain; agpaz@astrax.fis.ucm.es.

4 NASA/IPAC Extragalactic Database, California Institute of Technology, Pasadena, CA 91125; barry@ipac.caltech.edu.

5 Laboratoire d'Astrophysique de Marseille, BP 8, Traverse du Siphon, 13376 Marseille Cedex 12, France; samuel.boissier@oamp.fr, jose.donas@oamp.fr, bruno .milliard@oamp.fr.

6 Center for Astrophysical Sciences, Johns Hopkins University, Baltimore, MD 21218; dthilker@pha.jhu.edu,bianchi@pha.jhu.edu.

7 Instituto de Estructura de la Materia, CSIC, Serrano 121, Madrid 29006, Spain; carmen@damir.iem.csic.es.

8 California Institute of Technology, Pasadena, CA 91125; tab@srl.caltech .edu,tim@srl.caltech.edu,krl@srl.caltech.edu, friedman@srl.caltech.edu,cmartin@ srl.caltech.edu, patrick@srl.caltech.edu,ds@srl.caltech.edu, mseibert@srl.caltech .edu, tas@srl.caltech.edu,wyder@srl.caltech.edu.

${ }^{9}$ Laboratory for Astronomy and Solar Physics, NASA Goddard Space Flight Center, Greenbelt, MD 20771; neff@stars.gsfc.nasa.gov.

10 Department of Physics and Astronomy, UCLA, Los Angeles, CA 90095; rmr@astro.ucla.edu.

11 Department of Physics and Astronomy, Johns Hopkins University, Homewood Campus, Baltimore, MD 21218; heckman@pha.jhu.edu, szalay@pha.jhu .edu.

12 Center for Space Astrophysics, Yonsei University, Seoul 120-749, Korea; ywlee@csa.yonsei.ac.kr, yi@astro.ox.ac.uk.
}

This scenario, originally proposed to explain the color and metallicity gradients in the Milky Way, is also supported by recent $\mathrm{N}$-body/SPH simulations of the evolution of galactic disks (e.g., Brook et al. 2006).

Thus, star formation taking place now in some of the outermost regions of galaxies might probe physical conditions similar to those present during the formation of the first stars in the universe. The low gas densities found in these regions also provide an excellent test for the presence (or absence) of a threshold for star formation (Martin \& Kennicutt 2001) and for studying the behavior of the star formation law in low-density rarefied environments (Kennicutt 1989; Boissier et al. 2003, 2007; Elmegreen \& Hunter 2006).

Recent deep, wide-field observations of a sample of nearby spiral galaxies at UV wavelengths carried out by the Galaxy Evolution Explorer (GALEX) satellite as part of its Nearby Galaxies Survey (Bianchi et al. 2003; see also Gil de Paz et al. 2007) have revealed the presence of UV-bright complexes (XUV complexes hereafter) in the outermost parts of their disks. These galaxies, referred to as XUV disk galaxies, host UV emission well beyond ( 2-4 times) their optical (D25) radii (Thilker et al. 2005a; D. Thilker et al. 2007, in preparation; Gil de Paz et al. 2005). To date, the two best-studied XUV disks are those of M83 (Thilker et al. 2005a; $D=4.5 \mathrm{Mpc}$ ) and NGC 4625 (Gil de Paz et al. $2005 ; D=9.5 \mathrm{Mpc}$ ). These are two quite different examples of XUV disks: M83 has a very massive, large, high surface brightness optical disk with patchy XUV emission clearly disconnected from it; NGC 4625 is a low-luminosity system with ubiquitous XUV emission on top of an underlying low surface brightness optical disk (see Swaters \& Balcells 2002).

Any study of the properties of the XUV complexes discovered in M83 and NGC 4625 must establish whether the UV emission associated with these extended disks is due to recent star formation 
or not. If that is the case, as previous studies have suggested, a detailed analysis of their properties should then provide fundamental clues to understand the possible mechanism(s) that led to formation of stars in these outermost regions of the disks. These can be internal mechanisms, such as density waves, or external influences, such as tidal interactions or satellite disruption. Finally, we should be able to establish whether (1) this is continuously happening in the outer disks of these galaxies, (2) it is a transient but recurrent phenomenon that has taken place in these and other (perhaps all) spiral galaxies in the past, or, alternatively, (3) the XUV emission is a one-time phenomenon and these are the first generation of stars to form in the outer parts of these galaxies.

In order to gain some deeper insight into the properties of these XUV complexes, we have obtained deep optical spectroscopy of the outer disk of M83 and NGC 4625 using the Magellan and Palomar 200 inch $(5 \mathrm{~m})$ telescopes, respectively. In this work we study the physical conditions of the ionized gas (densities, metal abundances) in those XUV complexes showing $\mathrm{H} \alpha$ emission. We also estimate the properties of the ionizing sources by comparing the line ratios measured with the predictions of photoionization models; in particular, we determine if these line ratios are consistent with the line emission being due to photoionization by single stars as has been recently suggested (Gil de Paz et al. 2005).

The spectroscopic observations of the XUV disks of M83 and NGC 4625 along with the data reduction methodology are described in $\S 2$. In that section we also briefly describe complementary optical and GALEXUV imaging data. In $\S 3$ we present the results from the analysis of the spectroscopic data. We discuss the nature of the XUV emission and its possible implications for the past and future evolution of these systems in $\S 4$. The conclusions are summarized in $\S 5$.

\section{OBSERVATIONS AND REDUCTION}

\subsection{GALEX UV Imaging}

The regions analyzed here were originally identified in UV images taken by the GALEX satellite (Thilker et al. 2005a; Gil de Paz et al. 2005). GALEX is a NASA small explorer mission with a single instrument that consists of a Ritchey-Chrétien telescope with a $50 \mathrm{~cm}$ aperture that allows simultaneous imaging in two UV bands/channels, far-ultraviolet $\left(\mathrm{FUV} ; \lambda_{\text {eff }}=1516 \AA\right.$ ) and near-ultraviolet (NUV; $\lambda_{\text {eff }}=2267 \AA$ ), within a circular field of view of $1.2^{\circ}$ in diameter. See Martin et al. (2005) for a more detailed description of the mission and the GALEX instrument.

The GALEX observations used in this paper were carried out on 2003 June 7 in the case of M83 and on 2004 April 5 for NGC 4625. The total exposure times (equal in both the FUV and NUV bands) were 1352 and 1629 s for M83 and NGC 4625, respectively. These data were all reduced using the standard GALEX pipeline.

\subsection{Optical Imaging}

Ground-based optical imaging has been carried out to obtain accurate positions for the regions responsible for the XUV emission. Accuracies of the order of a few tenths of an arcsecond are needed for obtaining multiobject optical spectroscopy with relatively good spectral resolution for the $1^{\prime \prime}-1.5^{\prime \prime}$ wide slitlets used (see $\S 2.3$ ).

On 2005 March 10-14, we obtained deep broadband $U R$ imaging data of the southern part of the XUV disk of M83 using the $2048 \times 3150$ pixel Site 3 Direct CCD at the Cassegrain focus of the Swope $1 \mathrm{~m}$ telescope at Las Campanas Observatory (Chile) (see Fig. 1). The exposure time was $6 \times 1800 \mathrm{~s}$ in $U$ and $3 \times 900 \mathrm{~s}$

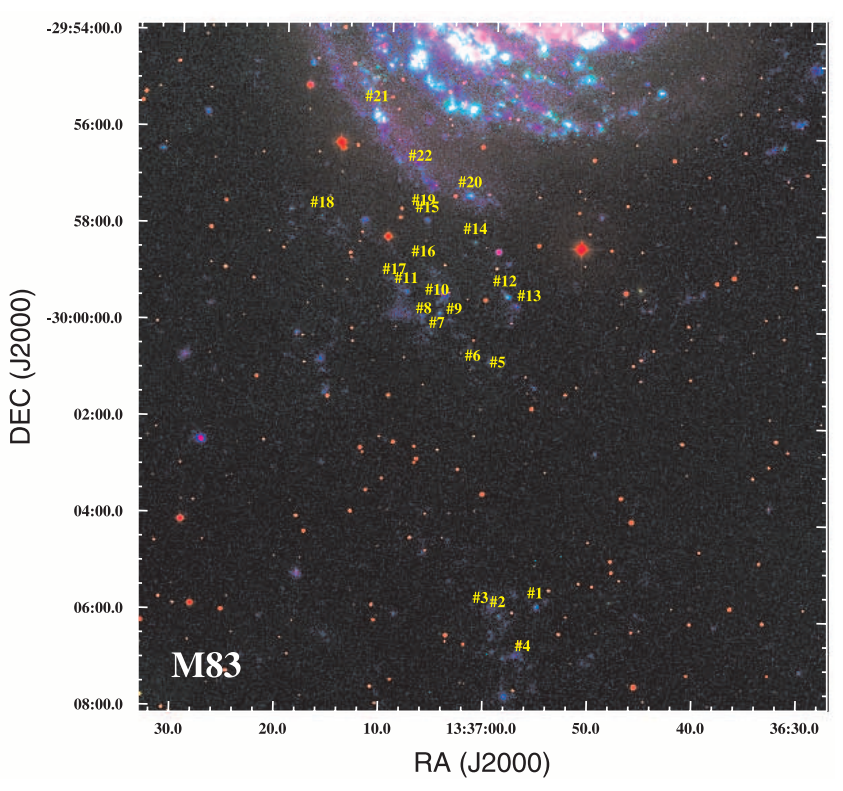

FIG. 1.-False-color RGB image of the southern region of the XUV disk of M83. This panel is a composition of the GALEX FUV (blue), ground-based continuum-subtracted $\mathrm{H} \alpha$ (green), and $U$-band (red) images. The $\mathrm{H} \alpha$-selected regions whose spectroscopy we present in this paper appear as green compact sources in this figure and are located right below the corresponding region identification number (see Table 2).

in $R$. Deep ground-based optical imaging of NGC 4625 in $B$ and $R$ bands had been previously obtained at the Isaac Newton $2.5 \mathrm{~m}$ telescope in La Palma (Spain) on 1995 May 28 and 1995 December 24 and 27 (see Fig. 2). Images are available through the Isaac Newton Group (ING) data archive. See also Gil de Paz et al. (2005) and Swaters \& Balcells (2002) for details on the optical imaging observations of NGC 4625 (see also Table 1).

Many of the galaxies with XUV-bright disks found by GALEX so far show a relatively sharp cutoff in the azimuthally averaged surface brightness profile in $\mathrm{H} \alpha$ at the limit of the optical disk, while the UV light apparently extends smoothly beyond that radius (Meurer et al. 2004; Thilker et al. 2005a; Gil de Paz et al. 2005). It is therefore expected that a significant fraction of the regions identified in the GALEX images would not show any line emission and, consequently, could not be used for determining the ionized gas metal abundances and dust reddening (from measurements of the Balmer line decrement). With this in mind and in order to improve the efficiency of our spectroscopic observations, we also obtained deep narrowband $\mathrm{H} \alpha$ imaging of both M83 and NGC 4625. M83 was observed on 2005 March 14 through a $70 \AA$ wide narrowband filter centered on $6570 \AA$ using the $2048 \times 3150$ pixel Site 3 Direct CCD attached to the Swope $1 \mathrm{~m}$ telescope in Las Campanas. The total exposure time in $\mathrm{H} \alpha$ was 10,200 s split in nine exposures. On 2004 August 20, we obtained an $800 \mathrm{~s}$ narrowband image of NGC 4625 through a $20 \AA$ wide filter centered on $6563 \AA$ using COSMIC mounted at the prime focus of the Palomar Observatory $5 \mathrm{~m}$ Hale telescope (Kells et al. 1998).

Reduction of the images was carried out using IRAF. ${ }^{13}$ For the $\mathrm{H} \alpha$ imaging data, the continuum was subtracted using a scaled $R$-band image such that the fluxes of the field stars in both images were equal. The astrometry of the optical images was carried out

13 IRAF is distributed by the National Optical Astronomy Observatory, which is operated by the Association of Universities for Research in Astronomy, Inc., under cooperative agreement with the National Science Foundation. 


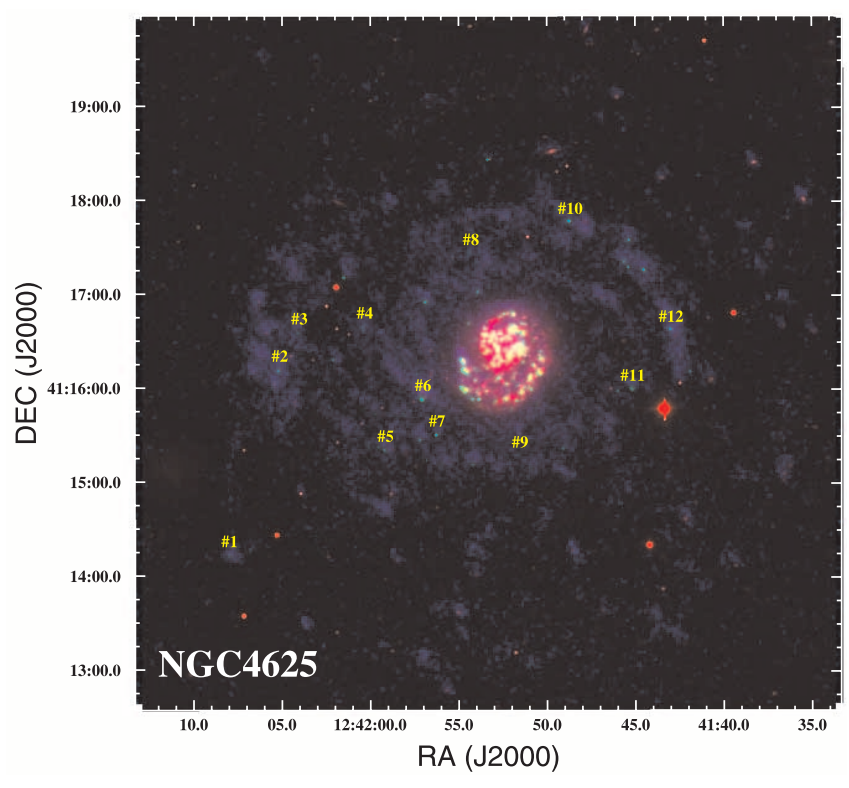

FIG. 2.-False-color RGB image of the XUV disk of NGC 4625. In this case we show a combination of the smoothed asinh-scaled FUV image (see Gil de Paz et al. 2005) (blue), the ground-based continuum-subtracted $\mathrm{H} \alpha$ image (green), and the $B$-band image (red). The $\mathrm{H} \alpha$-selected regions whose spectroscopy we present in this paper appear as green compact sources in this figure and are located right below the corresponding region identification number (see Table 2).

using the IRAF tasks starfind, ccxymatch, ccmap, and ccsetwcs. We made use of the USNO-B1 catalog in the case of M83 and the USNO-A2 catalog for the images of NGC 4625. The rms achieved by our astrometric calibration was smaller than $0.3^{\prime \prime}$ in all cases. The procedures followed for subtracting the continuum from the $\mathrm{H} \alpha$ images and for performing the astrometry of the optical images are described in detail in Gil de Paz et al. (2003).

False-color (RGB) composites using GALEX FUV, broadband, and narrowband ground-based optical images are shown in Figure 1 for M83 and in Figure 2 for NGC 4625. A summary of the UV and optical imaging observations is given in Table 1 .

\subsection{Optical Spectroscopy}

\subsubsection{Sample Selection}

Our spectroscopic sample is made up primarily of regions with detected emission in the continuum-subtracted narrowband images. The vast majority of these regions correspond to stellar associations that also show bright UV emission. In addition, we included $U$-bright sources with no obvious $\mathrm{H} \alpha$ counterpart, mainly to take advantage of the large field of view offered by the IMACS spectroscopic observations of M83. In this paper we focus exclusively on the analysis of the properties of the emissionline regions.

In Table 2 we give the coordinates of the emission-line regions in the XUV disks of M83 and NGC 4625. These are all the regions for which our follow-up spectroscopic observations confirmed both their nature as emission-line sources (both $\mathrm{H} \alpha$ and $\mathrm{H} \beta$ being detected in emission) and having a redshift close to that of the corresponding parent galaxy. The coordinates given in this table are accurate to the level given by the rms of our astrometric calibration, i.e., better than $0.3^{\prime \prime}$. Small systematic errors inherent to the USNO catalog could be also present.

\subsubsection{M83 Observations}

Multiobject spectroscopic observations of the southern region of the XUV disk of M83 (see Fig. 1) were obtained using the IMACS spectrograph at the $6.5 \mathrm{~m}$ Magellan-I (Baade) telescope in Las Campanas (Chile). The observations were carried out on 2005 April 18 using the $\mathrm{f} / 4$ long camera with the 300 line $\mathrm{mm}^{-1}$ grating in its first order. This configuration provides a spatial scale of $0.11^{\prime \prime}$ pixel $^{-1}$, a reciprocal dispersion of $0.743 \AA$ pixel $^{-1}$, and a spectral resolution of $R \sim 1200$ for slitlets of $1^{\prime \prime}$ in width. IMACS uses an $8 \mathrm{~K} \times 8 \mathrm{~K}$ pixel mosaic camera. The objects observed were distributed over two different masks with total exposure times of 40 and 60 minutes. During the night the seeing ranged between $0.6^{\prime \prime}$ and $1.0^{\prime \prime}$.

The spectra obtained were reduced using the so-called COSMOS package, an IMACS data reduction pipeline developed by August Oemler. COSMOS consists of a set of tasks that allow correcting the science images for bias and flat-fielding, subtracting the sky, and performing a precise mapping of the CCD pixels onto the coordinate system of wavelength and slit position using the observing setup and mask generation files as input.

In the case of the IMACS observations of M83 a total of $37 \mathrm{H} \alpha$-selected (34 in the XUV disk) and $32 U$-band-selected sources were included in the two masks observed. Out of the $37 \mathrm{H} \alpha$-selected sources, 22 of them (19 out of 34 in the XUV disk) were detected in both the $\mathrm{H} \alpha$ and $\mathrm{H} \beta$ lines in our spectroscopic data with redshifts close to that of M83. The positions and observed $\mathrm{H} \beta$ fluxes inside the slitlets for these 22 regions are given in Table 3. We also serendipitously discovered a background gal$\operatorname{axy}([G M B 2007] 1)$ at $z \simeq 0.3$ [R.A.(J2000.0) $=13^{\mathrm{h}} 37^{\mathrm{m}} 24.53^{\mathrm{s}}$,

TABLE 1

Summary of the Imaging Observations

\begin{tabular}{|c|c|c|c|c|c|c|c|}
\hline $\begin{array}{l}\text { Band } \\
\text { (1) }\end{array}$ & $\begin{array}{l}\text { Date } \\
(2)\end{array}$ & $\begin{array}{l}\text { Exposure } \\
\text { (s) } \\
\text { (3) }\end{array}$ & $\begin{array}{c}\text { Instrument } \\
\text { (4) }\end{array}$ & $\begin{array}{l}\text { Detector } \\
(5)\end{array}$ & $\begin{array}{l}\text { Telescope } \\
\text { (6) }\end{array}$ & $\begin{array}{c}\text { Scale } \\
\left(\operatorname{arcsec} \text { pixel }^{-1}\right) \\
(7)\end{array}$ & $\begin{array}{c}\text { Seeing } \\
(\operatorname{arcsec}) \\
(8)\end{array}$ \\
\hline \multicolumn{8}{|c|}{ M83 } \\
\hline$U \ldots \ldots \ldots \ldots$ & 2005 Mar $10-14$ & 10800 & Direct CCD & $2048 \times 3150$ SITe $3 \mathrm{CCD}$ & Swope $1 \mathrm{~m}$, LCO & 0.434 & 1.5 \\
\hline$R \ldots \ldots \ldots \ldots$ & 2005 Mar $10-14$ & 2700 & Direct CCD & $2048 \times 3150$ SITe $3 \mathrm{CCD}$ & Swope $1 \mathrm{~m}$, LCO & 0.434 & 1.1 \\
\hline $\mathrm{H} \alpha \ldots \ldots \ldots$ & 2005 Mar 14 & 10200 & Direct CCD & $2048 \times 3150$ SITe $3 \mathrm{CCD}$ & Swope $1 \mathrm{~m}, \mathrm{LCO}$ & 0.434 & 1.0 \\
\hline \multicolumn{8}{|c|}{ NGC 4625} \\
\hline$B \ldots \ldots \ldots \ldots$ & 1995 May 28 and Dec 24 & 2400 & PFCU & $1124 \times 1124$ TEK 3 CCD & INT 2.5 m, La Palma & 0.59 & 1.4 \\
\hline$R \ldots \ldots \ldots \ldots$ & 1995 May 28 and Dec 27 & 2200 & PFCU & $1124 \times 1124$ TEK 3 CCD & INT 2.5 m, La Palma & 0.59 & 1.3 \\
\hline $\mathrm{H} \alpha \ldots \ldots \ldots$ & 2004 Aug 20 & 800 & COSMIC & $2048 \times 2048$ SITe CCD & Hale $5 \mathrm{~m}$, Palomar & 0.40 & 1.0 \\
\hline
\end{tabular}


TABLE 2

XUV Region Parameters

\begin{tabular}{|c|c|c|c|c|c|}
\hline $\begin{array}{l}\text { XUV Region Name } \\
\text { (1) }\end{array}$ & $\begin{array}{c}\text { R.A. } \\
(\mathrm{J} 2000.0) \\
(2)\end{array}$ & $\begin{array}{c}\text { Decl. } \\
(\mathrm{J} 2000.0) \\
(3)\end{array}$ & $\begin{array}{c}f_{\mathrm{H} \beta} \\
\left(\mathrm{ergs} \mathrm{s}^{-1} \mathrm{~cm}^{-2} \text { ) }\right. \\
(4)\end{array}$ & $\begin{array}{c}f_{\mathrm{H} \beta, 0} \\
\left(\operatorname{ergs~s}^{-1} \mathrm{~cm}^{-2}\right) \\
(5)\end{array}$ & $\begin{array}{c}d \\
(\mathrm{kpc}) \\
(6)\end{array}$ \\
\hline \multicolumn{6}{|c|}{ M83 (NGC 5236) } \\
\hline NGC 5236: XUV $01 \ldots \ldots \ldots \ldots . . . . . .$. & 133655.149 & -300547.51 & $1.11 \times 10^{-15}$ & $1.11 \times 10^{-15}$ & 18.2 \\
\hline NGC 5236: XUV 02 ................. & 133658.720 & -300559.52 & $1.11 \times 10^{-16}$ & $2.39 \times 10^{-16}$ & 18.4 \\
\hline NGC 5236: XUV 03 ................. & 133700.371 & -300554.83 & $7.91 \times 10^{-17}$ & $2.07 \times 10^{-16}$ & 18.3 \\
\hline 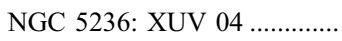 & 133656.222 & -300653.40 & $1.60 \times 10^{-16}$ & $1.60 \times 10^{-16}$ & 19.6 \\
\hline NGC 5236: XUV 05 ................. & 133659.284 & -300101.85 & $5.78 \times 10^{-17}$ & $3.33 \times 10^{-16}$ & 11.9 \\
\hline NGC 5236: XUV $06 \ldots \ldots \ldots \ldots . . . . . . .$. & 133701.676 & -300054.14 & $5.52 \times 10^{-17}$ & $5.52 \times 10^{-17}$ & 11.7 \\
\hline NGC 5236: XUV 07 .................. & 133705.182 & -300014.37 & $7.06 \times 10^{-17}$ & $3.02 \times 10^{-16}$ & 10.9 \\
\hline NGC 5236: XUV 08 .................. & 133706.470 & -295956.73 & $3.97 \times 10^{-17}$ & $5.02 \times 10^{-17}$ & 10.6 \\
\hline NGC 5236: XUV 09 .................. & 133705.001 & -295946.05 & $1.07 \times 10^{-15}$ & $1.57 \times 10^{-15}$ & 10.3 \\
\hline 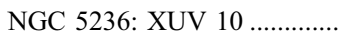 & 133705.242 & -295933.30 & $3.28 \times 10^{-16}$ & $4.57 \times 10^{-16}$ & 10.0 \\
\hline 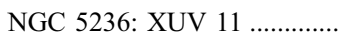 & 133708.196 & -295919.67 & $4.05 \times 10^{-16}$ & $4.05 \times 10^{-16}$ & 9.9 \\
\hline NGC 5236: XUV $12 \ldots \ldots \ldots \ldots . . . .$. & 133658.772 & -295920.82 & $1.85 \times 10^{-16}$ & $9.46 \times 10^{-16}$ & 9.7 \\
\hline NGC 5236: XUV $13 \ldots \ldots \ldots \ldots . . . .$. & 133658.523 & -295924.54 & $3.70 \times 10^{-15}$ & $6.93 \times 10^{-15}$ & 9.8 \\
\hline NGC 5236: XUV $14 \ldots \ldots \ldots \ldots . . .$. & 133701.713 & -295816.75 & $2.18 \times 10^{-16}$ & $6.67 \times 10^{-16}$ & 8.3 \\
\hline NGC 5236: XUV $15 \ldots \ldots \ldots \ldots . . .$. & 133706.354 & -295751.15 & $1.97 \times 10^{-16}$ & $1.97 \times 10^{-16}$ & 7.9 \\
\hline 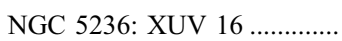 & 133706.627 & -295846.02 & $9.70 \times 10^{-17}$ & $2.48 \times 10^{-16}$ & 9.1 \\
\hline 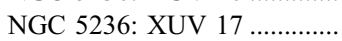 & 133709.362 & -295908.94 & $9.12 \times 10^{-17}$ & $1.42 \times 10^{-16}$ & 9.7 \\
\hline NGC 5236: XUV $18 \ldots \ldots \ldots \ldots$ & 133716.414 & -295748.10 & $4.02 \times 10^{-16}$ & $7.32 \times 10^{-16}$ & 8.8 \\
\hline NGC 5236: XUV 19 & 133706.730 & -295742.46 & $4.74 \times 10^{-17}$ & $8.60 \times 10^{-17}$ & 7.7 \\
\hline NGC 5236: XUV $20^{\mathrm{a}} \ldots \ldots \ldots \ldots$ & 133702.316 & -295718.89 & $1.80 \times 10^{-15}$ & $7.10 \times 10^{-15}$ & 7.0 \\
\hline NGC 5236: XUV $21^{\mathrm{a}} \ldots \ldots \ldots \ldots . . . . .$. & 133711.409 & -295534.79 & $3.24 \times 10^{-15}$ & $1.18 \times 10^{-14}$ & 5.6 \\
\hline NGC 5236: XUV 22 & 133707.142 & -295647.37 & $1.24 \times 10^{-15}$ & $3.00 \times 10^{-15}$ & 6.6 \\
\hline \multicolumn{6}{|c|}{ NGC 4625} \\
\hline NGC 4625: XUV $01 \ldots . . . \ldots \ldots . . . .$. & 124207.985 & +411413.65 & $1.33 \times 10^{-16}$ & $3.75 \times 10^{-16}$ & 10.0 \\
\hline 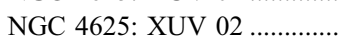 & 124205.136 & +411612.14 & $4.10 \times 10^{-16}$ & $1.14 \times 10^{-15}$ & 6.5 \\
\hline 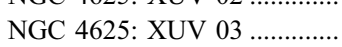 & 124204.025 & +411635.98 & $1.73 \times 10^{-16}$ & $3.71 \times 10^{-16}$ & 5.9 \\
\hline 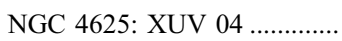 & 124200.322 & +411639.80 & $1.66 \times 10^{-16}$ & $9.95 \times 10^{-16}$ & 4.0 \\
\hline 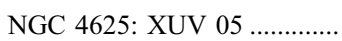 & 124159.150 & +411521.11 & $2.05 \times 10^{-16}$ & $3.20 \times 10^{-16}$ & 4.5 \\
\hline 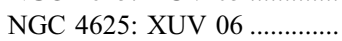 & 124157.029 & +411553.65 & $6.75 \times 10^{-16}$ & $1.57 \times 10^{-15}$ & 2.7 \\
\hline NGC 4625: XUV $07 \ldots \ldots \ldots \ldots . . . . .$. & 124156.234 & +411530.90 & $4.66 \times 10^{-16}$ & $1.21 \times 10^{-15}$ & 3.1 \\
\hline 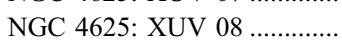 & 124154.310 & +411726.82 & $1.61 \times 10^{-16}$ & $7.11 \times 10^{-16}$ & 2.9 \\
\hline 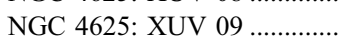 & 124151.538 & +411517.52 & $1.02 \times 10^{-16}$ & $2.78 \times 10^{-16}$ & 3.2 \\
\hline NGC 4625: XUV $10 \ldots \ldots \ldots$ & 124148.677 & +411746.81 & $1.01 \times 10^{-15}$ & $1.01 \times 10^{-15}$ & 4.4 \\
\hline 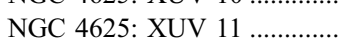 & 124145.151 & +411600.29 & $2.60 \times 10^{-16}$ & $2.35 \times 10^{-15}$ & 4.3 \\
\hline 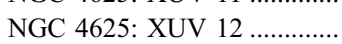 & 124142.977 & +411637.95 & $5.33 \times 10^{-16}$ & $1.10 \times 10^{-15}$ & 3.7 \\
\hline
\end{tabular}

Notes.- - Units of right ascension are hours, minutes, and seconds, and units of declination are degrees, arcminutes, and arcseconds. Col. (1): Region identification. Col. (2): Right ascension. Col. (3): Declination. Col. (4): Observed $\mathrm{H} \beta$ flux inside the corresponding slitlet. Col. (5): Extinction-corrected $\mathrm{H} \beta$ flux inside the slitlet; color excesses are given in Table 3. Col. (6): Galactocentric distance in kpc.

${ }^{\text {a }}$ Region belongs to the optical disk of M83.

decl.(J2000.0) $\left.=-29^{\circ} 57^{\prime} 20.1^{\prime \prime}\right]$ whose emission in [O III] 25007 falls in the window defined by the filter used in our narrowband imaging observations and for which we also detected $\mathrm{H} \beta$ and [O III] $\lambda 4959$.

\subsubsection{NGC 4625 Observations}

The spectroscopic observations of the XUV disk of NGC 4625 were carried out using COSMIC mounted at the prime focus of the Palomar Observatory $5 \mathrm{~m}$ (Hale) telescope. The spectra were taken on the night of 2005 March 16 using the 300 line $\mathrm{mm}^{-1}$ grating and a mask with $1.5^{\prime \prime}$ wide slitlets. COSMIC uses a single $2048 \times 2048$ pixel CCD detector. The total exposure time of the single mask observed was 40 minutes, and the seeing during the observations was $\sim 1.2^{\prime \prime}$. The spectra were reduced using standard IRAF tasks within the CCDRED, ONEDSPEC, and TWODSPEC packages.
Out of the $17 \mathrm{H} \alpha$-selected targets included in the mask, 12 sources showed emission in at least $\mathrm{H} \alpha$ and $\mathrm{H} \beta$ at the redshift of NGC 4625 and were used in our analysis (see Table 3 for the list of positions and observed fluxes in $\mathrm{H} \beta$ ). In addition, we identified a background galaxy ([GMB2007]2) at $z \simeq 0.3$ located at R.A.(J2000.0) $=12^{\mathrm{h}} 41^{\mathrm{m}} 41.89^{\mathrm{s}}$ and decl.(J2000.0) $=$ $+41^{\circ} 16^{\prime} 10.9^{\prime \prime}$.

\subsubsection{Flux Calibration}

The spectroscopic observations of M83 and NGC 4625 were both made under photometric conditions. In the case of M83 we observed the spectroscopic standard star LTT 3218 using a $2.5^{\prime \prime}$ wide long-slit mask. We adopted the extinction curve for Cerro Tololo Inter-American Observatory. For the observations of NGC 4625 we obtained spectra of BD +33 2642 and HD 93521 using a 4" wide long-slit mask. The extinction 
TABLE 3

XUV REgion Line Ratios

\begin{tabular}{|c|c|c|c|c|c|c|c|c|c|c|c|}
\hline $\begin{array}{l}\text { XUV Region Name } \\
\text { (1) }\end{array}$ & $\begin{array}{c}E(B-V) \\
\quad(2)\end{array}$ & $\begin{array}{c}{\left[\begin{array}{l}\mathrm{O} \\
\mathrm{III}\end{array}\right] /[\mathrm{O} \mathrm{II}]} \\
\text { (3) }\end{array}$ & $\begin{array}{c}{[\mathrm{O} \quad \mathrm{III}] / \mathrm{H} \beta} \\
\quad(4)\end{array}$ & $\begin{array}{c}{[\mathrm{N} \mathrm{II]} / \mathrm{H} \alpha} \\
(5)\end{array}$ & $\begin{array}{c}{\left[\mathrm{S}_{\mathrm{II}}\right] / \mathrm{H} \alpha} \\
(6)\end{array}$ & $\begin{array}{l}6717 / 6731 \\
(7)\end{array}$ & $\begin{array}{l}\mathrm{R} 23 \\
(8)\end{array}$ & $\begin{array}{l}\log \mathrm{EW}_{\mathrm{H} \alpha} \\
\text { (9) }\end{array}$ & $\begin{array}{l}\log \mathrm{EW}_{[\mathrm{O} \mathrm{m}]} \\
\quad(10)\end{array}$ & $\begin{array}{c}Z_{l / h, \mathrm{M} 91} \\
(11)\end{array}$ & $\begin{array}{l}Z_{l / h, \mathrm{PT} 05} \\
\text { (12) }\end{array}$ \\
\hline \multicolumn{12}{|c|}{ M83 (NGC 5236) } \\
\hline NGC 5236: XUV 01 ................. & 0.00 & 0.38 & 0.49 & -0.89 & -1.11 & 1.79 & 0.76 & 2.71 & 2.28 & $7.861 / 8.680$ & $7.718 / 8.458$ \\
\hline NGC 5236: XUV 02 .................. & 0.23 & $>0.76$ & 0.86 & -1.24 & $<-0.73$ & $\ldots$ & $<1.05$ & $>1.80$ & $>1.91$ & $\ldots$ & $\ldots$ \\
\hline NGC 5236: XUV 03 .................. & 0.29 & $>-0.20$ & 0.14 & -0.65 & $<-0.47$ & $\ldots$ & $<0.68$ & 2.89 & 2.12 & $\ldots$ & $\ldots$ \\
\hline NGC 5236: XUV 04 ................. & 0.00 & & $<-0.21$ & -0.66 & $<-0.11$ & $\ldots$ & $<0.30$ & 2.23 & $<2.00$ & . & $\ldots$ \\
\hline NGC 5236: XUV 05 ................... & 0.53 & $<-0.42$ & $<-0.06$ & -0.37 & -0.50 & 1.04 & $<0.63$ & 2.51 & $<3.10$ & $\ldots$ & $\ldots$ \\
\hline NGC 5236: XUV 06 ................. & 0.00 & $<-0.52$ & $<0.01$ & -0.51 & -0.65 & 0.88 & $<0.76$ & 2.96 & $<1.53$ & $\ldots$ & $\ldots$ \\
\hline NGC 5236: XUV 07 ................. & 0.44 & -0.73 & -0.07 & -0.60 & -0.98 & 2.40 & 0.85 & 2.56 & 1.76 & $8.391 / 8.427$ & 7.765/7.744 \\
\hline NGC 5236: XUV 08 ................ & 0.07 & $<-0.78$ & $<0.12$ & $\ldots$ & $<-0.19$ & $\ldots$ & $<1.09$ & 2.75 & $<1.64$ & & $\ldots$ \\
\hline NGC 5236: XUV 09 ................. & 0.12 & 0.06 & 0.39 & -0.89 & -0.88 & 2.29 & 0.79 & 3.38 & 2.67 & $8.015 / 8.617$ & $7.891 / 8.316$ \\
\hline NGC 5236: XUV $10 \ldots \ldots \ldots \ldots . . . .$. & 0.10 & -0.28 & 0.03 & -0.81 & -0.76 & 1.11 & 0.62 & 2.99 & 2.10 & $7.862 / 8.761$ & $7.506 / 8.341$ \\
\hline NGC 5236: XUV 11 .................. & 0.00 & 0.17 & 0.48 & -1.02 & -1.11 & 2.01 & 0.83 & 2.52 & 2.42 & $8.049 / 8.584$ & $7.952 / 8.311$ \\
\hline NGC 5236: XUV 12 ................ & 0.49 & -0.02 & 0.16 & -0.52 & -0.84 & 1.07 & 0.59 & 2.72 & 2.34 & $7.730 / 8.798$ & $7.550 / 8.492$ \\
\hline NGC 5236: XUV 13 ................. & 0.19 & 0.04 & 0.32 & -0.69 & -1.01 & 1.21 & 0.72 & 3.39 & 3.29 & $7.904 / 8.689$ & $7.751 / 8.391$ \\
\hline NGC 5236: XUV $14 \ldots \ldots \ldots \ldots . . . .$. & 0.34 & -0.29 & -0.13 & -0.46 & -0.74 & 0.87 & 0.47 & 2.63 & 1.63 & $7.660 / 8.875$ & $7.306 / 8.468$ \\
\hline NGC 5236: XUV 15 .................. & 0.00 & $>-0.56$ & -0.66 & -0.39 & -0.60 & 0.77 & $<0.14$ & 2.88 & 1.90 & $\ldots$ & $\ldots$ \\
\hline NGC 5236: XUV 16 ................ & 0.28 & $<-0.78$ & $<-0.46$ & -0.34 & -0.72 & 1.60 & $<0.51$ & $>1.91$ & $<0.81$ & $\ldots$ & $\ldots$ \\
\hline NGC 5236: XUV 17 ................. & 0.13 & -0.58 & 0.05 & -0.85 & -0.65 & 0.83 & 0.86 & 3.03 & 1.93 & $8.348 / 8.442$ & $7.877 / 7.824$ \\
\hline NGC 5236: XUV 18 ................. & 0.18 & .. & 0.13 & -0.48 & -0.93 & 1.06 & $\ldots$ & 2.85 & 3.10 & 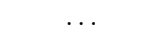 & $\ldots$ \\
\hline NGC 5236: XUV 19 .................. & 0.18 & $\ldots$ & $<0.03$ & -0.43 & -0.31 & 0.97 & $<0.74$ & $>1.41$ & $<1.84$ & $\ldots$ & $\ldots$ \\
\hline NGC 5236: XUV $20 \ldots \ldots \ldots \ldots \ldots \ldots . . . .$. & 0.41 & -0.49 & -0.54 & -0.38 & $<-1.82$ & $\ldots$ & 0.20 & 2.76 & 1.23 & $\ldots / 9.007$ & $\ldots / 8.543$ \\
\hline NGC 5236: XUV 21 ................ & 0.39 & -0.29 & -0.59 & -0.38 & -0.93 & 1.31 & 0.01 & 3.05 & 1.78 & $\ldots / 9.061$ & $\ldots / 8.696$ \\
\hline NGC 5236: XUV 22 .................. & 0.27 & 0.02 & -0.27 & -0.38 & -0.78 & 1.30 & 0.14 & $>2.67$ & 3.54 & $\ldots / 9.026$ & $\ldots / 8.759$ \\
\hline \multicolumn{12}{|c|}{ NGC 4625} \\
\hline NGC 4625: XUV 01 ................ & 0.31 & 0.51 & 0.59 & -1.26 & $<-0.59$ & $\ldots$ & 0.83 & 2.85 & 2.54 & $7.941 / \ldots$ & $7.787 / \ldots$ \\
\hline NGC 4625: XUV 02 .................. & 0.31 & -0.43 & 0.30 & -1.05 & -0.94 & 1.90 & 0.99 & 2.76 & 2.24 & $8.531 / \ldots$ & $8.486 / \ldots$ \\
\hline NGC 4625: XUV 03 ................. & 0.23 & $\ldots$ & $<-0.23$ & $\ldots$ & -0.51 & 1.63 & $<0.69$ & 2.82 & $<2.28$ & $\ldots$ & $\ldots$ \\
\hline NGC 4625: XUV $04 \ldots \ldots \ldots \ldots \ldots . . .$. & 0.54 & $<-0.99$ & $<-0.23$ & -0.91 & -0.63 & 1.19 & $<0.92$ & 2.77 & $<1.65$ & & $\ldots$ \\
\hline NGC 4625: XUV 05 .................. & 0.13 & -0.25 & 0.22 & -0.81 & -0.79 & 0.90 & 0.79 & 2.37 & 2.00 & $8.123 / \ldots$ & $7.868 / \ldots$ \\
\hline NGC 4625: XUV 06 ................. & 0.25 & -0.65 & -0.07 & -0.81 & -0.66 & 0.68 & 0.79 & 2.57 & 1.68 & $8.265 / \ldots$ & $7.635 / \ldots$ \\
\hline NGC 4625: XUV 07 .................... & 0.29 & -0.09 & 0.44 & -1.09 & -1.04 & 1.20 & 0.92 & $>2.32$ & $>2.07$ & $8.296 / \ldots$ & $8.269 / \ldots$ \\
\hline NGC 4625: XUV 08 ................. & 0.45 & $\ldots$ & 0.52 & -0.99 & $<-0.69$ & $\ldots$ & $\ldots$ & 3.22 & 2.63 & & $\ldots$ \\
\hline NGC 4625: XUV 09 ................. & 0.30 & 0.25 & 0.96 & -0.85 & $<-0.44$ & $\ldots$ & 1.28 & 3.02 & 3.16 & $8.400 / \ldots$ & $8.400 / \ldots$ \\
\hline NGC 4625: XUV 10 ................... & 0.00 & $\ldots$ & 0.68 & -1.29 & -0.66 & 1.36 & .. & 3.12 & 2.95 & $\ldots$ & $\ldots$ \\
\hline NGC 4625: XUV $11 \ldots \ldots \ldots \ldots . . .$. & 0.66 & $>0.26$ & 0.92 & -1.18 & $<-0.68$ & $\ldots$ & $<1.24$ & 2.61 & $>2.04$ & & $\ldots$ \\
\hline NGC 4625: XUV 12 ................... & 0.22 & -0.55 & 0.07 & -1.10 & -0.69 & 1.73 & 0.85 & 3.07 & 2.32 & $8.333 / \ldots$ & $7.891 / \ldots$ \\
\hline
\end{tabular}

Notes.-Col. (1): Region identification. Col. (2): Color excess derived from the $\mathrm{H} \alpha / \mathrm{H} \beta$ Balmer line decrement; it includes the effects of both the Galactic and the internal dust extinction. Col. (3): Logarithm of the extinction-

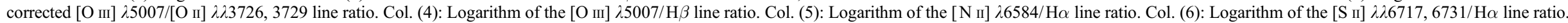

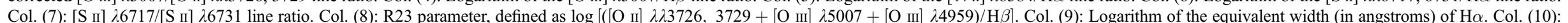

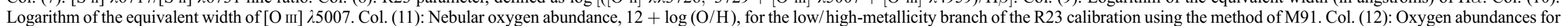
the low/high-metallicity branch of the R23 calibration using the method of PT05. We have adopted $12+\log (\mathrm{O} / \mathrm{H})_{\odot}=8.69$ throughout the text (Allende Prieto et al. 2001). 

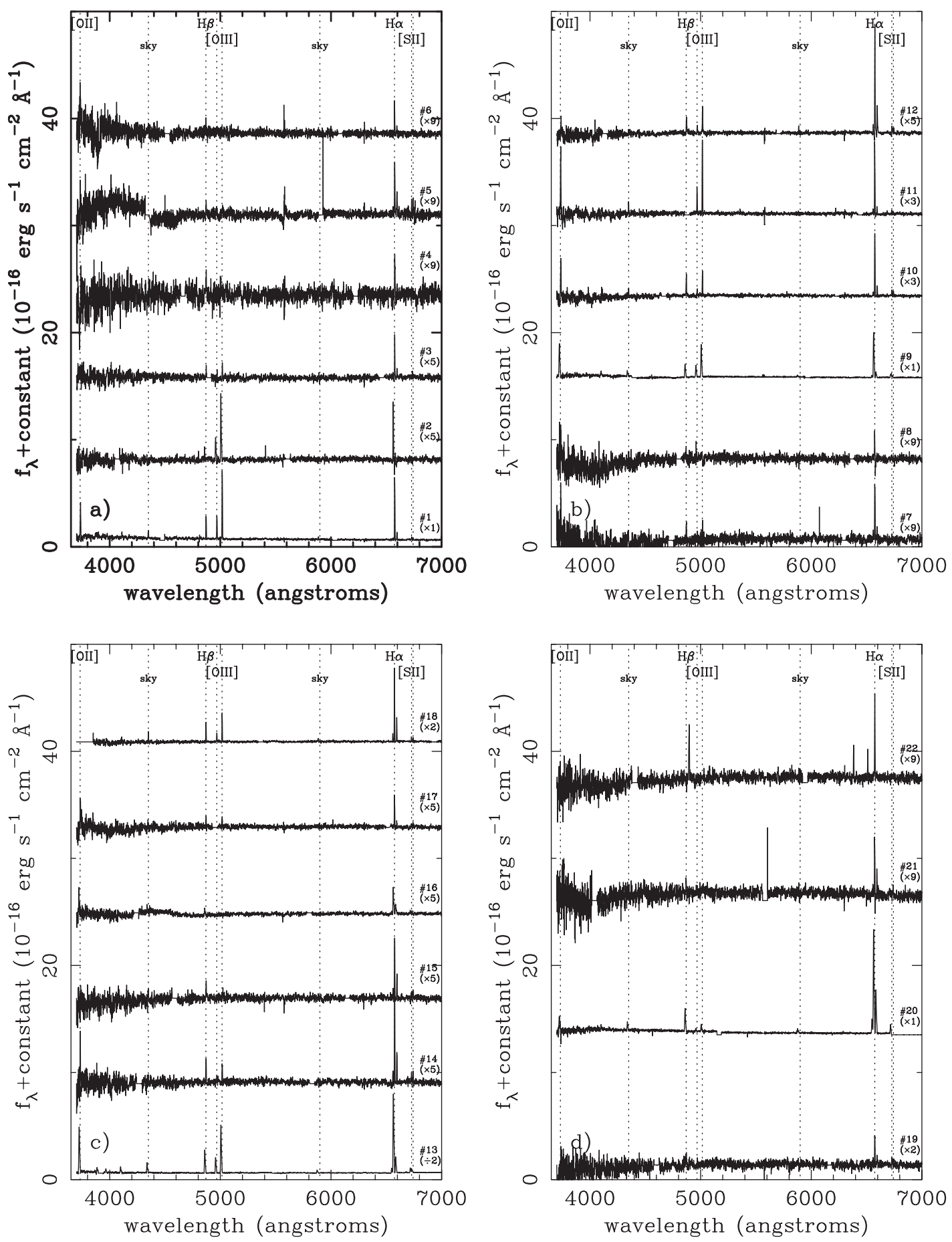

Fig. 3.- Individual spectra of $\mathrm{H} \alpha$-selected regions in the XUV disk of M83, plus three regions located in its optical disk (NGC 5236: XUV 20, 21, and 22). Only regions with detected $\mathrm{H} \alpha$ emission at the approximate redshift of M83 are shown. Identification number (according to Table 2) and scaling factors are shown.

curve of Kitt Peak National Observatory was adopted in this case.

The flux-calibrated spectra obtained for the 22 (12) $\mathrm{H} \alpha$-selected regions in M83 (NGC 4625) used for this work are shown in Figure 3 (Fig. 4). Since the observation of the spectrophotometric standard stars was performed near the center of the field, we compared the resulting flux-calibrated sky spectra obtained through each of the slitlets in order to ensure the validity of this calibration for all sources in the mask.

For M83 the absolute calibration shows a dispersion of $\sim 0.3$ mag (Fig. 5a). However, if we apply systematic offsets to the individ- ual spectra (which do not affect the line ratios measured), the dispersion between these spectra due to changes in the wavelength dependence of the flux calibration is reduced to $\sim 0.15$ mag between 4000 and $6000 \AA$ and $\sim 0.2$ mag below $4000 \AA$ and above $6000 \AA$ (Fig. $5 b$ ). These numbers represent the average $1 \sigma$ calibration errors for our spectra.

In the case of NGC 4625 both the absolute and relative differences between all the sky spectra extracted are significantly smaller than for M83 (see Figs. $5 c$ and $5 d$ ). Once small global offsets are applied, the rms between the spectra becomes $\sim 0.1 \mathrm{mag}$ for $\lambda<4300 \AA$ or $\lambda>6600 \AA$ or even smaller for 


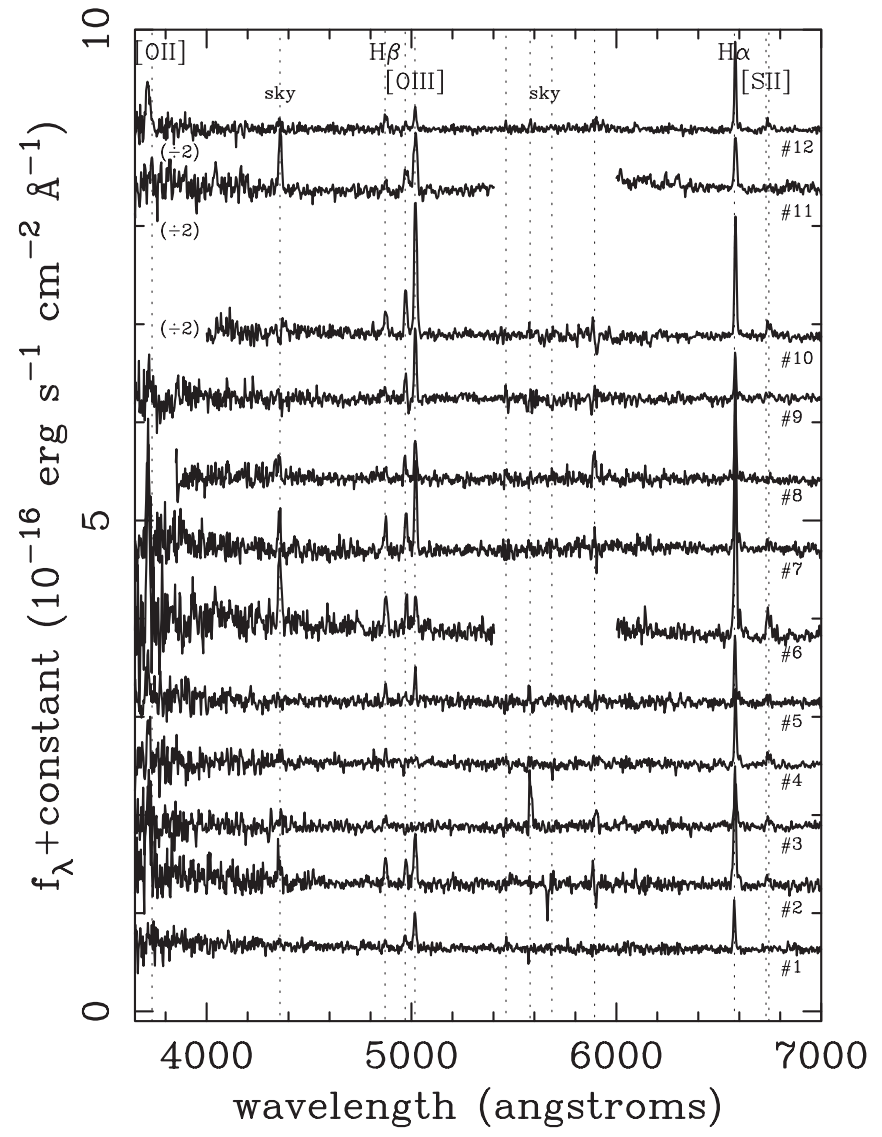

FIG. 4.- Individual spectra of $\mathrm{H} \alpha$-selected regions in the XUV disk of NGC 4625. Only regions with detected $\mathrm{H} \alpha$ emission at the approximate redshift of NGC 4625 are shown. Identification number (according to Table 2) and scaling factors are shown. Spectral regions with high sky subtraction residuals in the spectra of NGC 4625: XUV 06 and NGC 4625: XUV 11 have been blanked out for the sake of clarity.

$4300 \AA<\lambda<6600 \AA$. This is probably due to the much reduced field of view of this instrument and smaller format of the detector.

\section{RESULTS}

\subsection{Line Fluxes and Ratios}

The extinction-corrected $\mathrm{H} \beta$ line fluxes measured through our slitlets are given in Table 2. These spectroscopic fluxes are all below the value expected for a region ionized by a single $\mathrm{O} 3 \mathrm{star}$ ( $L_{\mathrm{H} \alpha} \simeq 10^{38} \mathrm{ergs} \mathrm{s}^{-1}$; Sternberg et al. 2003) at the distance of our targets. Similar conclusions were reached by Gil de Paz et al. (2005) through the analysis of narrowband imaging data on NGC 4625. Note that although many of these regions are very compact, aperture effects due to the limited size of our slitlets $\left(1^{\prime \prime}-1.5^{\prime \prime}\right.$ in width) might be significant. Therefore, the spectroscopic line fluxes given in Table 2 should be considered as lower limits to the total observed flux of the corresponding $\mathrm{H}$ II region.

In Table 3 we present the line ratios measured for each of the 19 (12) confirmed line-emitting regions in the XUV disk of M83 (NGC 4625), plus three additional regions in its optical disk (NGC 5236: XUV 20, 21, and 22). Both fluxes and line ratios have been corrected for foreground Galactic and internal extinction using the $\mathrm{H} \alpha / \mathrm{H} \beta$ Balmer line decrement. We have adopted an intrinsic value for $\mathrm{H} \alpha / \mathrm{H} \beta$ of 2.86 for case $\mathrm{B}$ recombination (Osterbrock 1989) and the parameterization of the Galactic extinction law given by Cardelli et al. (1989).
Figure 6 shows the standard diagnostic diagrams proposed by Baldwin et al. (1981) and Veilleux \& Osterbrock (1987). The line ratios measured in the XUV disk of M83 (Figs. $6 a$ and $6 b$ ) are similar to those found in local star-forming galaxies (SFGs), represented here by the $\mathrm{H} \alpha$-selected UCM Survey galaxies of Zamorano et al. (1994, 1996). We find that regions in the inner (optical) disk of M83 $\left(r<5.5^{\prime}\right.$; filled circles) have lower excitations than those in the inner part of the XUV disk $\left(5.5^{\prime}<\right.$ $r<10^{\prime}$; filled triangles) and even lower than in the outermost XUV disk $\left(r>10^{\prime}\right.$; filled stars $)$.

On other hand, the line-emitting regions in the XUV disk of NGC 4625 show relatively high excitation conditions. We also find a larger dispersion in the $[\mathrm{O} \mathrm{III}] \lambda 5007 / \mathrm{H} \beta$ ratio for a given value of $[\mathrm{N}$ II $] \lambda 6584 / \mathrm{H} \alpha$ than in the case of M83. Gil de Paz et al. (2005) showed that many of the regions identified in the XUV disk of NGC 4625 have $\mathrm{H} \alpha$ luminosities consistent with photoionization due to single massive stars. Interestingly, simultaneous low [O III] $\lambda 5007 / \mathrm{H} \beta$ and [N II] $\lambda 6584 / \mathrm{H} \alpha$ ratios are predicted by some of the single-star photoionization models of Stasinska \& Schaerer (1997). These models do not cover the entire parameter space of the properties of the ionizing star (luminosity, metallicity, etc.) or the surrounding H II region (density, geometry), nor were they intended for reproducing the line ratios of single-star $\mathrm{H}$ II regions but their ionization structure. Thus, a detailed modeling of all the observed line ratios for each individual region covering a wider range of parameters than those explored by Stasinska \& Schaerer (1997) is carried out in $\S 3.3$ (see also Fig. 6).

Figures $6 b$ and $6 d$ show the predictions of the photoionization models for evolving starbursts developed by Stasinska \& Leitherer (1996). In Figure $6 b$ the models represent the variation in the line ratios with age between 1 and $10 \mathrm{Myr}$ for different metallicities $\left(Z_{\odot}, Z_{\odot} / 4, Z_{\odot} / 10, Z_{\odot} / 40\right)$. For starburst models with metallicities below solar the sequence in excitation conditions seen in these figures is mainly due to a change in age, from young high-excitation starbursts (whose effective temperature is dominated by very massive stars) at the top left part of the diagram to more evolved low-excitation starbursts that can be found at the bottom right.

The fact that the loci of the emission-line XUV complexes in these diagrams coincide with those of local star-forming galaxies indicates that the line emission detected is produced by photoionization by young massive stars and not by planetary nebulae, which typically show lower [O II] $\lambda 3727 / \mathrm{H} \beta$ and somewhat higher [O III $] \lambda 5007 / \mathrm{H} \beta$ line ratios than those found here (see, e.g., Baldwin et al. 1981). The compact nature of these sources (see Figs. 1 and 2) also excludes the possibility of this line emission being associated with diffuse ionized gas (DIG).

\subsection{Metal Abundances Based on Strong Lines}

Determining the metallicity of the ionized gas in star-forming galaxies and $\mathrm{H}$ II regions generally means measuring their oxygen abundances. Ideally this requires deriving the electron temperature from the measurement of a temperature-sensitive line ratio such as [O III] $\lambda 4363 /[\mathrm{O}$ III] $\lambda 5007$. Unfortunately, the [O III] $\lambda 4363$ line is very weak and can only be detected in bright, actively star-forming low-metallicity systems.

Since the [O III] $\lambda 4363$ line is not detected in our spectra, in order to constrain the metallicities of the emission-line regions in the XUV disks of M83 and NGC 4625, we rely on spectral diagnostics based on strong lines such as those described in Kewley $\&$ Dopita (2002). Among these, perhaps the best estimator of the nebular oxygen abundance is the R23 ratio. Defined as $\log \left[\left([\mathrm{O}\right.\right.$ II $\left.\left.] \lambda 3727+\left[\mathrm{O}_{\mathrm{III}}\right] \lambda 5007+\left[\mathrm{O}_{\mathrm{III}}\right] \lambda 4959\right) / \mathrm{H} \beta\right]$, the $\mathrm{R} 23$ 

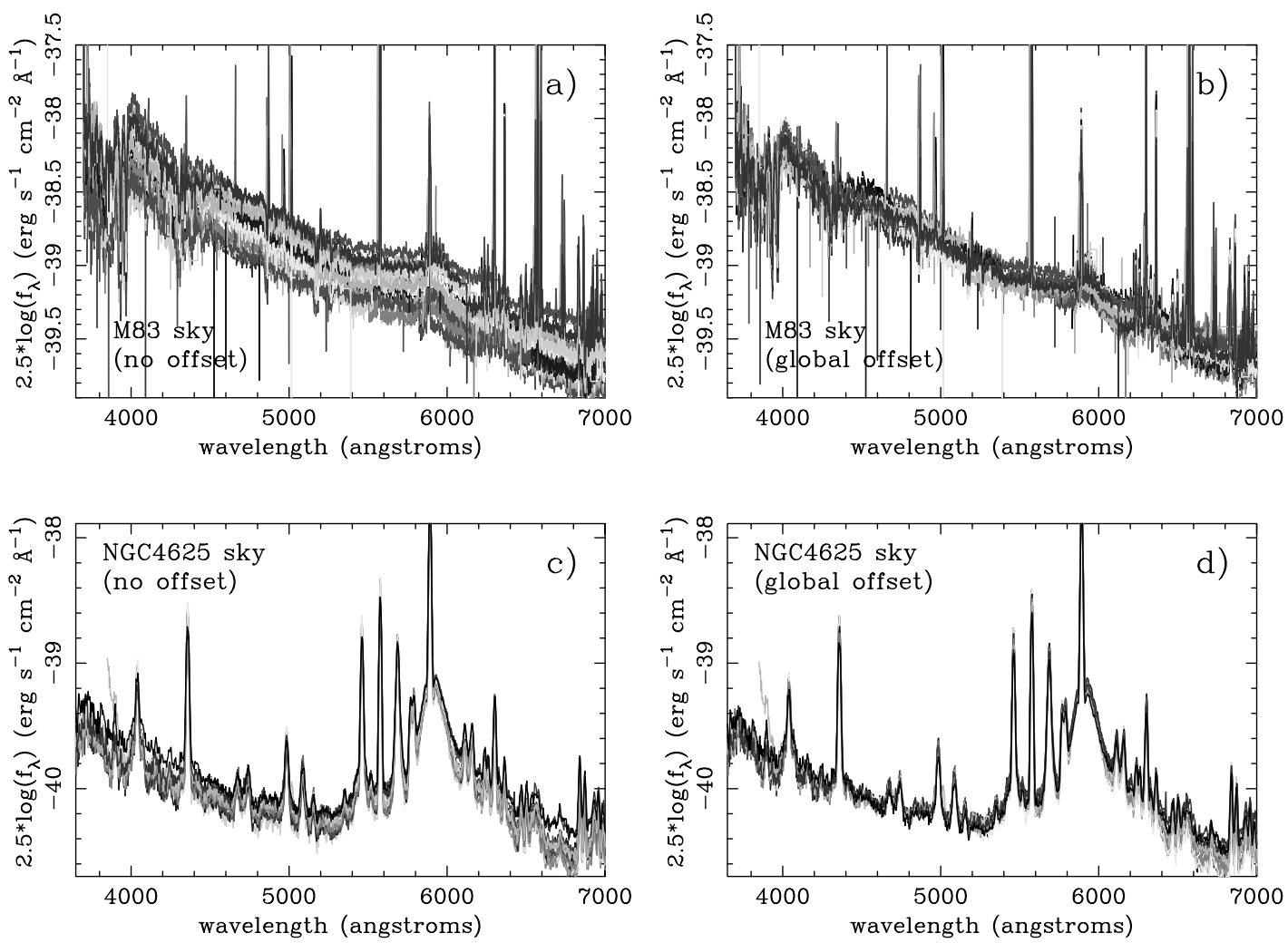

FIG. 5.- (a) Flux-calibrated sky (+object) spectra within each of the slitlets obtained as part of the spectroscopic observations of the XUV disk of M83 (different

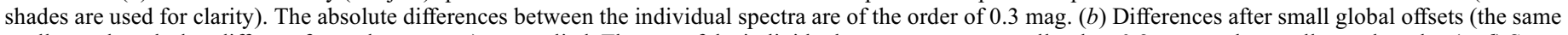

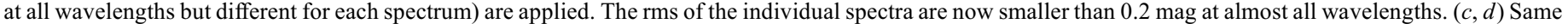
as $(a, b)$, but for the observations of NGC 4625. [See the electronic edition of the Journal for a color version of this figure.]

parameter was first introduced by Pagel et al. (1979). Although very sensitive to metal abundance, this parameter is also sensitive to the ionization parameter, especially at low metallicities $\left(Z<Z_{\odot}\right)$. This degeneracy between metallicity and ionization parameter can be partially solved by taking into account the value of an indicator mostly sensitive to the ionization parameter, such as the [O II] $\lambda \lambda 3726,3729 /[\mathrm{O}$ III] $\lambda 5007$ (hereafter $[\mathrm{O}$ II]/[O III]) ratio (e.g., McGaugh 1991, hereafter M91).

Another critical issue regarding the use of the $\mathrm{R} 23$ ratio is the fact that it is double valued for most R23 flux ratios. It shows a maximum that, depending on the ionization parameter, is located anywhere between half-solar and solar metallicity, and below that maximum the value of the R 23 can be reproduced by either a low-metallicity or a high-metallicity photoionization model. In order to solve this problem, an initial guess on the oxygen abundance based on some other diagnostics or secondary indicators, such as the $\left[\mathrm{N}_{\text {II }}\right] \lambda 6584 /\left[\mathrm{O}_{\text {II }}\right] \lambda \lambda 3726,3729\left(\left[\mathrm{~N}_{\text {II }}\right] /\left[\mathrm{O}_{\text {II }}\right]\right),\left[\mathrm{N}_{\text {II }}\right] \lambda 6584 /\left[\mathrm{S}_{\text {II }}\right]$ $\lambda \lambda 6717,6731\left(\left[\mathrm{~N} \mathrm{II}_{\mathrm{II}}\right] /[\mathrm{S} \mathrm{II}]\right)$, or $\left[\mathrm{N}_{\mathrm{II}}\right] \lambda 6584 / \mathrm{H} \alpha([\mathrm{N} \mathrm{II}] / \mathrm{H} \alpha)$ ratios, is desirable.

We have used two different recipes, those published by M91 and Pilyugin \& Thuan (2005, hereafter PT05), which allow for a determination of the oxygen abundance as a simple function of the R23 ratio and the $\left[\mathrm{O}_{\mathrm{II}}\right] /\left[\mathrm{O}_{\mathrm{III}}\right]$ line ratio, once either the low- or highmetallicity branch is chosen. Note that for high metallicities only the method of PT05 includes a dependence on the $[\mathrm{O}$ II $] /[\mathrm{O}$ III $]$ line ratio through the use of the excitation parameter, $P[\equiv([\mathrm{O}$ III $] \lambda 5007+$ $[\mathrm{O}$ III $] \lambda 4959) /\left([\mathrm{O}\right.$ II $] \lambda 3727+[\mathrm{O}$ III $\left.] \lambda 5007+\left[\begin{array}{ll}\mathrm{O} & \mathrm{III}\end{array} \lambda 4959\right)\right]$.

\subsection{1. $M 83$}

The large dispersion in the line ratios measured across the disk of M83 indicates a wide range of ionization conditions and/or metal abundances. Therefore, we should be very cautious in choosing what branch of the parameterization of the R23 ratio to use.

In the case of the three innermost regions analyzed in this paper (NGC 5236: XUV 20, 21, and 22), which are still considered part of the optical disk of the galaxy, the $[\mathrm{N}$ II $] /[\mathrm{O}$ II $]$ ratios measured are high enough $\left[\log \left(\left[\mathrm{N}_{\mathrm{II}}\right] /[\mathrm{O} \mathrm{II}]\right)>0\right.$ in all three cases $]$ that we can confidently assume the high-metallicity branch for the calibration of the R23 parameter. For the regions in the XUV disk of $\mathrm{M} 83$ the $[\mathrm{N} \mathrm{II}] /[\mathrm{O}$ II] ratios measured (between -1.1 and -0.3 in log scale) indicate metal abundances around solar. The $[\mathrm{N}$ II $] /[\mathrm{S}$ II $]$ ratios measured, ranging between -0.2 and 0.4 in logarithmic scale but with most of the regions showing positive values, favor again metal abundances close to solar. In the case of the $[\mathrm{N} \mathrm{II}] / \mathrm{H} \alpha$ line ratios, whose values range approximately between -1 and -0.4 , we can only say that metallicities are probably above $Z_{\odot} / 2$. We should point out that all [N $\left.\mathrm{N}_{\mathrm{II}}\right] /[\mathrm{O}$ II], $[\mathrm{N}$ II $] /[\mathrm{S}$ II $]$, and $[\mathrm{N}$ II $] / \mathrm{H} \alpha$ are good diagnostics for the oxygen abundance as long as the N/O abundance ratio adopted by Kewley \& Dopita (2002) is appropriate for these regions (see below for further discussion). These numbers do not enable us to determine which of the two branches of the calibration of the R23 ratio with metallicity should be used in the case of the emission-line regions of the XUV disk of M83. Accordingly, in Table 3 we give the values derived assuming both the low- and high-metallicity cases for both the M91 and PT05 calibrations. The fact that these secondary indicators suggest metal abundances close to the limit between the low- and high-metallicity branches of the R23 calibration results in very similar oxygen abundance estimates under the two assumptions for regions NGC 5236: XUV 07 and NGC 5236: XUV 17. 

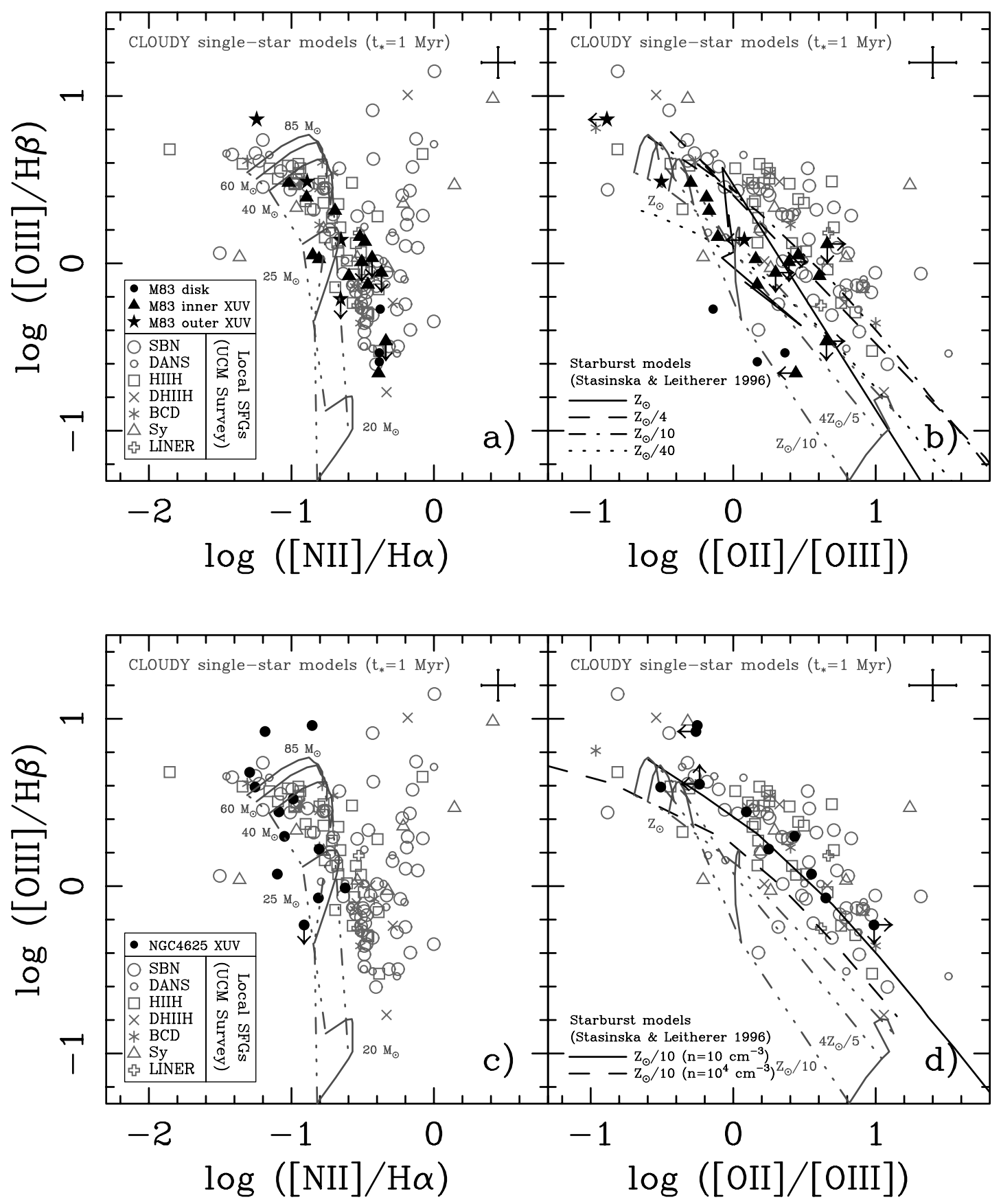

FIG. 6.-Diagnostic diagrams for the emission-line regions in the XUV disks of M83 and NGC 4625. (a) [O III] $\lambda 5007 / \mathrm{H} \beta$ vs. [ $\mathrm{N}$ II] $\lambda 6584 / \mathrm{H} \alpha$ extinction-corrected line ratios. The data for M83 are coded based on their galactocentric distance: regions in the inner (optical) disk $\left(r<5.5^{\prime}\right.$; filled circles), regions in the inner XUV disk $\left(5.5^{\prime}<r<10^{\prime}\right.$; filled triangles), and regions in the outer XUV disk $\left(r>10^{\prime}\right.$; filled stars). For comparison we show the location of the UCM Survey galaxies, a complete sample of local SFGs and active galactic nuclei selected by their emission in $\mathrm{H} \alpha$ (Gallego et al. 1996), coded by spectroscopic type. The lines are the predictions of Cloudy single-star photoionization models for an age of the star of $1 \mathrm{Myr}$ and a solar N/O abundance ratio (see $\S 3.3$ ). Solid lines correspond to models with fixed mass for the ionizing star and metallicities ranging between $Z_{\odot} / 10$ and $Z_{\odot}$, while dot-dashed lines correspond to models with fixed metallicity and different masses between 20 and $85 M_{\odot}$. Typical errors on the line ratios are shown at the top right corner of the diagram. $(b)[\mathrm{O}$ III] $\lambda 5007 / \mathrm{H} \beta \mathrm{vs}$. [O II] $\lambda \lambda 3726,3729 /[\mathrm{O}$ III $\lambda 25007 \mathrm{extinction}$-corrected line ratios. Here we also show the predictions of photoionization models for evolving starbursts (Stasinska \& Leitherer 1996), with different lines showing the predictions for different metallicities (see text for more details on the models). (c) Same as (a), but for NGC 4625. (d) Same as (b), but for NGC 4625. The starburst photoionization models shown here correspond to $1 / 10$ solar abundance models with two different densities, 10 and $10^{4} \mathrm{~cm}^{-3}$. [See the electronic edition of the Journal for a color version of this figure.]

The R23 calibration of PT05 yields systematically lower oxygen abundances than those derived using the M91 method (similar systematic differences have been found by van Zee \& Haynes [2006] when comparing the calibrations of M91 and Pilyugin [2000]). This is the case for both the lower and upper branch of the calibration. These differences range from $\leq 0.1$ dex in regions NGC 5236: XUV 01 and 09 and NGC 4625: XUV 01 and 07 to $\geq 0.4$ dex in regions NGC 5236: XUV 07 and 17 and NGC 4625: XUV 06. The largest differences between the predictions of these two methods are found in regions located in the so-called transition zone (PT05). It is worth mentioning that the lower (upper) branch of the empirical calibration of PT05 was limited to oxygen abundances $12+\log (\mathrm{O} / \mathrm{H})<8.00[12+\log (\mathrm{O} / \mathrm{H})>8.25]$ and values of the excitation parameter $0.55<P<1(P>0.1)$. 
Thus, oxygen abundances in Table 3 not fulfilling these criteria are extrapolated values and should be considered with caution.

The photoionization models of Kewley \& Dopita (2002) assume an N/O abundance ratio that is approximately constant [ $\log (\mathrm{N} / \mathrm{O}) \sim-1.5$; i.e., nitrogen is assumed of primary origin] at low oxygen abundances $[12+\log (\mathrm{O} / \mathrm{H})<8.5]$ and rises linearly with the oxygen abundance above this value (i.e., secondary nitrogen). They used an empirical fit to the abundance measurements of van Zee et al. (1998a). However, the N/O ratio is highly dependent on the actual stellar yields, star formation and gas infall history of individual galaxies, and on the initial mass function (IMF; Prochaska et al. 2002; Pilyugin et al. 2003; Köppen \& Hensler 2005; Mollá et al. 2006). In the case of the XUV disks these properties may be different from those found in the $\mathrm{H}$ II regions of actively star-forming dwarfs and optical spiral disks, places where metal abundances are traditionally measured. For example, according to some of the scenarios proposed to explain the lack of line emission in XUV disks (Meurer et al. 2004), the IMF in these regions might have a lack of very massive stars. Such a top-light IMF would result in both the oxygen and nitrogen being released by intermediate-mass stars, which would lead to a high N/O abundance ratio, higher than that adopted by Kewley \& Dopita (2002) at low oxygen abundances. Should the actual N/O abundance ratio in XUV disks behave differently from what these authors assume, some of the conclusions drawn above from the analysis of secondary indictors could change. A more extensive discussion on the possible $\mathrm{N} / \mathrm{O}$ abundance ratios in these XUV disks is given in $\S$ 3.3.2.

Moreover, some of the conclusions on the analysis of the secondary oxygen abundance indicators are valid only if the ionization parameter in these regions is in the range considered by standard photoionization models such as those of Kewley \& Dopita (2002). Here we should remind the reader that it has not been proved yet that photoionization models of typical H II regions adequately reproduce the physical conditions present in these, newly discovered, XUV disks.

This paper is a first attempt to study the physical properties of the ionized gas in these regions, so we face many limitations that are inherent to previous empirical and theoretical studies mainly focused on the analysis of $\mathrm{H}$ II regions in the main body of spiral and irregular galaxies. In particular, the ionization parameter in the $\mathrm{H}$ II regions of the XUV disks could be either significantly higher or lower than in Galactic $\mathrm{H}$ in regions (due, for example, to a hotter/cooler mean stellar population). They could also show a different density structure than that found in typical $\mathrm{H}$ II regions and perhaps similar to that of the disks of low surface brightness (LSB) galaxies, where the lower interstellar medium (ISM) pressure is thought to result in a more simple density structure (Mihos et al. 1999). Moreover, single-star photoionization models might be more appropriate when trying to reproduce the conditions and output spectra of these regions (see $\S 3.3$ ). And finally, the N/O abundance ratio in XUV disks may be different from that measured elsewhere (see $\S$ 3.3.2).

\subsubsection{NGC 4625}

For NGC 4625 the $\left[\mathrm{N}_{\mathrm{II}}\right] /\left[\mathrm{O}_{\mathrm{II}}\right]$ ratios in the emission-line regions of its XUV disk are all below or very close to -1.0 (in logarithmic scale), with the highest value being -0.94 . Therefore, according to the photoionization models of Kewley \& Dopita (2002), the metallicities are necessarily below solar (for any ionization parameter between $5 \times 10^{6}$ [or lower] and $3 \times 10^{8} \mathrm{~cm} \mathrm{~s}^{-1}$ ). We remind the reader that we are using $12+\log (\mathrm{O} / \mathrm{H})_{\odot}=8.69$ (Allende Prieto et al. 2001). Moreover, the $B$-band luminositymetallicity relationship recently derived by Salzer et al. (2005) indicates that for the luminosity of NGC $4625\left(M_{B} \simeq-17.4 \mathrm{mag}\right)$ the vast majority of the local star-forming galaxies show oxygen abundances clearly below $12+\log (\mathrm{O} / \mathrm{H})<8.5$.

Therefore, it is reasonable to conclude that the low-metallicity branch should be adopted for the XUV disk of NGC 4625, which is bluer and probably less chemically evolved than the overall galaxy stellar population (Gil de Paz et al. 2005). It is worth emphasizing here that the $\left[\mathrm{N}_{\mathrm{II}}\right] /\left[\mathrm{O}_{\mathrm{II}}\right]$ ratio depends only weakly on the ionization parameter. This conclusion would still be valid even if the $\mathrm{N} / \mathrm{O}$ abundance ratio in these regions is higher than $\log (\mathrm{N} / \mathrm{O})=-1.5$, the constant value adopted by Kewley $\&$ Dopita (2002) for primary nitrogen production.

In one of the regions of the XUV disk of NGC 4625 (NGC 4625: XUV 09), the R23 value derived is somewhat larger than the maximum value of this parameter for the corresponding $[\mathrm{O} \mathrm{II}] /[\mathrm{O} \mathrm{III}]$ ratio (or excitation parameter, $P$ ), which results in an abnormally high value for the oxygen abundance, in spite of its low $[\mathrm{N} \mathrm{II}] /[\mathrm{O}$ II] ratio $(-1.2$ in the $\log )$. This suggests that in this region the metal abundance is probably close to the value for which the R23 ratio is maximum according to both photoionization models and empirical recipes $[12+\log (\mathrm{O} / \mathrm{H}) \simeq 8.4$ for $\log ([\mathrm{O} \mathrm{II}] /[\mathrm{O} \mathrm{III}]) \simeq 0$; see, e.g., Kobulnicky et al. 1999] and that observational errors might have moved its value above this maximum. Therefore, in the particular case of NGC 4625: XUV 09 we adopt $12+\log (\mathrm{O} / \mathrm{H})=8.4$ as our best metallicity estimate. The PT05 calibration is of limited use in the case of NGC 4625, since all regions analyzed in its XUV disk (except for NGC 4625: XUV 01) show excitation parameters, $P$, well below 0.55 , the minimum value given by PT05 as giving reliable results. Its PT05based oxygen abundances are, consequently, extrapolated values.

\subsection{Photoionization Modeling}

\subsubsection{Physical Parameters of the Models}

In order to study the physical conditions in the XUV disks, we have compared the observed line ratios with the predictions of a complete set of $\sim 13,000$ photoionization models covering a wide range of physical properties both for the gas and for the source of ionizing radiation. Calculations were performed with version 06.02 of Cloudy, last described by Ferland et al. (1998).

First, we ran two sets of 3262 simulations each using single massive stars from the CoStar library of Schaerer \& de Koter (1997) with zero-age main-sequence (ZAMS) masses 20, 25, 40, 60,85 , and $120 M_{\odot}$, ages between 1 and $7 \mathrm{Myr}$ (or the lifetime of the star, whichever happens first) with a step of 1 Myr for stars $>25 M_{\odot}$ and of 2 Myr below that mass, and two different stellar metallicities $\left(Z_{\odot}\right.$ and $\left.Z_{\odot} / 5\right)$. The luminosity of the star was determined using the relations given by Tout et al. (1996). For the gas we adopted a spherical distribution with constant density 1, 10, $10^{2}$, and $10^{3} \mathrm{~cm}^{-3}$ and an inner radius between $10^{16}$ and $10^{17.5} \mathrm{~cm}$ (in steps of $0.5 \mathrm{dex})$. The outer radius was set to $10^{21} \mathrm{~cm}(\sim 325 \mathrm{pc})$ in order to ensure that all modeled $\mathrm{H}$ II regions were ionization bounded. The metal abundance of both gas and dust grains was allowed to simultaneously vary between $Z_{\odot} / 40$ and $2 Z_{\odot}$, with values $1 / 40,1 / 20,1 / 10,1 / 5,3 / 10,2 / 5,1 / 2,3 / 5,4 / 5,1,1.5$, and $2 Z_{\odot}$. The first set of realizations was run adopting a constant solar $\mathrm{N} / \mathrm{O}$ abundance ratio $\left[\log (\mathrm{N} / \mathrm{O})_{\odot}=-0.76\right]$, while for the second one we adopted a constant value of $\log (\mathrm{N} / \mathrm{O})=-1.5$ for oxygen abundances below $12+\log (\mathrm{O} / \mathrm{H})=8.45$ and an $\mathrm{N} / \mathrm{O}$ rising linearly with $\mathrm{O} / \mathrm{H}$ above that value, i.e., a behavior similar to that adopted in the photoionization models developed by Kewley \& Dopita (2002). We stopped the calculation when the gas temperature reached $3000 \mathrm{~K}$, the value at which the contribution of the gas to the optical line emission is negligible. 
As we commented in $\S 3.1$, the weak-line fluxes of these H II regions indicate that they are most likely powered by one single massive star, at a maximum a few, but not by a massive stellar cluster. In order to further verify this result, we also ran two sets of models (with two different N/O abundance ratios) of 3456 Cloudy realizations each where the gas was ionized by a stellar cluster with a mass of $10^{3} M_{\odot}$ (see Gil de Paz et al. 2005). For the stellar continuum we used the predictions of the Starburst 99 population synthesis models (Leitherer et al. 1999) for metallicities between $Z_{\odot} / 20$ and $2 Z_{\odot}$ (with values $1 / 20,1 / 5,2 / 5,1$, and $2 Z_{\odot}$, i.e., those of the Geneva tracks used in these realizations) and ages in the range 1-9 Myr (with steps of $0.1 \mathrm{Myr}$ ), assuming the IMF of Kroupa et al. (1993). We made use of the Starburst99 default (recommended) stellar atmospheres of Pauldrach/Hillier (Pauldrach 2005; Hillier 2003; Lejeune et al. 1997). The spatial distribution and chemical composition of the gas and dust grains were those used for the single-star photoionization realizations described above. We used a somewhat finer grid of gas densities ( 0.5 dex steps instead of $1 \mathrm{dex})$ in order to better explore the ionization parameter. Thanks to the homology relations of photoionization models with the ionization parameter, the use of a fixed stellar mass for the cluster should not strongly limit the range of possible line ratios derived as long as the density of the gas is adequately sampled.

For $\sim 10 \%$ of the starburst models the outer radius $\left(10^{21} \mathrm{~cm}\right.$, i.e., $325 \mathrm{pc}$ ) was reached before the temperature was below $3000 \mathrm{~K}$. This happens only for very low densities $\left(10^{0.0}-10^{0.5} \mathrm{~cm}^{-3}\right)$ and only for low gas metallicities or for high metallicities when the ionizing starburst is young ( $\leq 3 \mathrm{Myr}$ ). None of the best-fitting solutions derived correspond to one of these "density-bounded" models.

The best-fitting set of models in each case was determined by $\chi^{2}$ minimization of the extinction-free line intensities normalized to $\mathrm{H} \beta$ in logarithmic scale. We adopted an average error of $0.10 \mathrm{dex}$ in all line ratios, except for [O II] $\lambda \lambda 3726,3729 / \mathrm{H} \beta$ and [S II] $\lambda \lambda 6717,6731 / \mathrm{H} \beta$, where errors of 0.15 and 0.20 dex were assumed, respectively (see $\S 2.3 .4$ ). We checked that the intrinsic $\mathrm{H} \alpha / \mathrm{H} \beta$ ratio predicted by these realizations was similar (within $\sim 5 \%$ ) to the case B recombination coefficient assumed as part of our procedure for correcting the observed line ratios for extinction. Only the properties of regions for which the $\left[\mathrm{O}_{\mathrm{II}}\right] \lambda \lambda 3726$, $3729 / \mathrm{H} \beta$, [O III] $\lambda 5007 / \mathrm{H} \beta$, and [ $\left.\mathrm{N}_{\text {III }}\right] \lambda 6584 / \mathrm{H} \beta$ line ratios are available were determined. When the $\left[\mathrm{S}_{\text {II }}\right] \lambda \lambda 6717,6731 / \mathrm{H} \beta$ line ratio was available, we included it also in the minimization. We note that the $\mathrm{S} / \mathrm{O}$ abundance ratio is approximately constant with the oxygen abundance and similar to the solar value (see Kehrig et al. 2006 and references therein).

\subsubsection{N/O Abundance Ratio}

The N/O abundance ratio is a function of the stellar yields, the star formation and gas infall histories of galaxies, and of the IMF. Most galaxies follow a relatively well defined sequence in the $\mathrm{N} / \mathrm{O}-\mathrm{O} / \mathrm{H}$ diagram with a constant value of the N/O ratio for oxygen abundances below $12+\log (\mathrm{O} / \mathrm{H})=8.5$, which suggests a primary origin for the nitrogen and an $\mathrm{N} / \mathrm{O}$ increasing linearly with $\mathrm{O} / \mathrm{H}$ above that value, which is explained in terms of a secondary production of nitrogen (van Zee et al. 1998a; Liang et al. 2006; for a theoretical review on the subject see also Mollá et al. 2006). However, as we commented in $\S 3.2 .1$, both the history and IMF of the outermost regions of spiral disks (such as those analyzed here) may be quite different from those of the galaxies where nitrogen and oxygen abundances have been traditionally measured. Due to this limitation, we decided to consider two possible N/O abundance ratios in our photoionization models: one similar to that used by Kewley \& Dopita (2002) and that is based on the results of van Zee et al. (1998a), and the other where $\mathrm{N} / \mathrm{O}$ is constant throughout the entire range in oxygen abundance and equal to the solar value. The main difference between the predictions of these two sets of models is the intensity of the [N II] $\lambda 6584$ line. This is due to the fact that this line or other nitrogen lines have only a small contribution to the cooling of the gas, so a change in the N/O abundance ratio does not alter significantly the temperature structure of the nebula.

Tables 4-7 show that in the case of M83 the models where the $\mathrm{N} / \mathrm{O}$ is assumed to be constant and equal to the solar value provide a better agreement with the data than those obtained using an $\mathrm{O} / \mathrm{H}$-scaled $\mathrm{N} / \mathrm{O}$ abundance ratio. The observed increase in the best-fitting reduced $\chi^{2}$ for M83 is a consequence of the [N II] $\lambda 6584$ line in the models being too weak for any set of physical parameters of the nebula compatible with the observed oxygen line ratios. This can be clearly seen in Table 8, where we compare the observed line ratios (in logarithmic scale) with those predicted by the best-fitting photoionization model obtained for each combination of ionization source and N/O ratio. The models with $\mathrm{O} / \mathrm{H}$-scaled $\mathrm{N} / \mathrm{O}$ abundance ratio systematically underpredict the value of the extinction-corrected [N $\left.\mathrm{N}_{\mathrm{II}}\right] \lambda 6584 / \mathrm{H} \beta$ line ratio.

This result suggests that the actual N/O abundance ratio in the XUV disk of M83 might be higher than that obtained in actively star-forming dwarf galaxies and in the optical disks of spiral galaxies at low oxygen abundances $[\log (\mathrm{N} / \mathrm{O})=-1.5]$. There are several possible explanations for such a high N/O, e.g., the presence of a dominant evolved stellar population (Pilyugin et al. 2003; Mollá et al. 2006) or a top-light IMF. The former scenario seems unlikely considering the relatively blue colors measured in the outer parts of these and other spiral disks (Muñoz-Mateos et al. 2007; see also $\S 4.3$ ). Alternatively, an episodic star formation history with the last episode of star formation taking place several Gyr ago (i.e., long enough for the release of nitrogen by intermediate-mass stars) could also result in relatively high N/O ratios.

Another possible scenario involves the infall of pristine gas in regions with moderate (close to solar) oxygen abundance where the nitrogen production is already secondary. This gas infall would drive the system into a region of lower oxygen abundance without changing the N/O ratio (Köppen \& Hensler 2005). This scenario has been also proposed to explain the large dispersion in the N/O ratio at oxygen abundances $12+\log (\mathrm{O} / \mathrm{H}) \geq 7.9$ (including some objects with high values of N/O) recently found in a sample of dwarf irregular galaxies with low ionization parameters (van Zee \& Haynes 2006).

In the case of NGC 4625 the results obtained for the two different N/O ratios considered are comparable except for region NGC 4625: XUV 05, where the solar N/O yields a better fit, and for regions NGC 4625: XUV 09 and 12, where an $\mathrm{O} / \mathrm{H}$-scaled $\mathrm{N} / \mathrm{O}$ ratio is favored. However, for most regions (all except NGC 4625: XUV 01) the line fluxes predicted by the best-fitting singlestar models with an $\mathrm{O} / \mathrm{H}$-scaled $\mathrm{N} / \mathrm{O}$ ratio are systematically lower than the lower limits measured by up to a factor of 40 (see Tables 2 and 5).

For the remainder of the paper we focus on the results obtained for the set of models with a solar N/O abundance ratio (Tables 4 and 6) since they provide a better fit to the data overall.

\subsubsection{Best-fitting Single-Star Models}

In Table 4 we show the parameters of the best-fitting singlestar photoionization model with a solar N/O ratio. We find that the lowest reduced $\chi^{2}$ achieved are not very different from 1 for most 
TABLE 4

Best-fitting Single-Star Photoionization Models (Solar N/O Ratio)

\begin{tabular}{|c|c|c|c|c|c|c|c|c|c|}
\hline $\begin{array}{l}\text { XUV Region Name } \\
\text { (1) }\end{array}$ & $\begin{array}{l}\chi^{2} \\
(2)\end{array}$ & $\begin{array}{c}f_{\mathrm{H} \beta, \text { model }} \\
\left(\mathrm{ergs} \mathrm{s}^{-1} \mathrm{~cm}^{-2} \text { ) }\right. \\
\text { (3) }\end{array}$ & $\begin{array}{l}\mathrm{Age}_{*} \\
(\mathrm{Myr}) \\
(4)\end{array}$ & $\begin{array}{c}T_{\text {eff, } *} \\
(\mathrm{~K}) \\
(5)\end{array}$ & $\begin{array}{c}\log g_{*} \\
(6)\end{array}$ & $\begin{array}{c}\operatorname{Mass}_{*} \\
\left(M_{\odot}\right) \\
(7)\end{array}$ & $\begin{array}{c}Z_{\text {gas }} \\
\left(Z_{\odot}\right) \\
(8)\end{array}$ & $\begin{array}{c}\log n_{\text {gas }} \\
\left(\mathrm{cm}^{-3}\right) \\
(9)\end{array}$ & $\begin{array}{c}\log R_{\text {in }} \\
(\mathrm{cm}) \\
(10)\end{array}$ \\
\hline
\end{tabular}

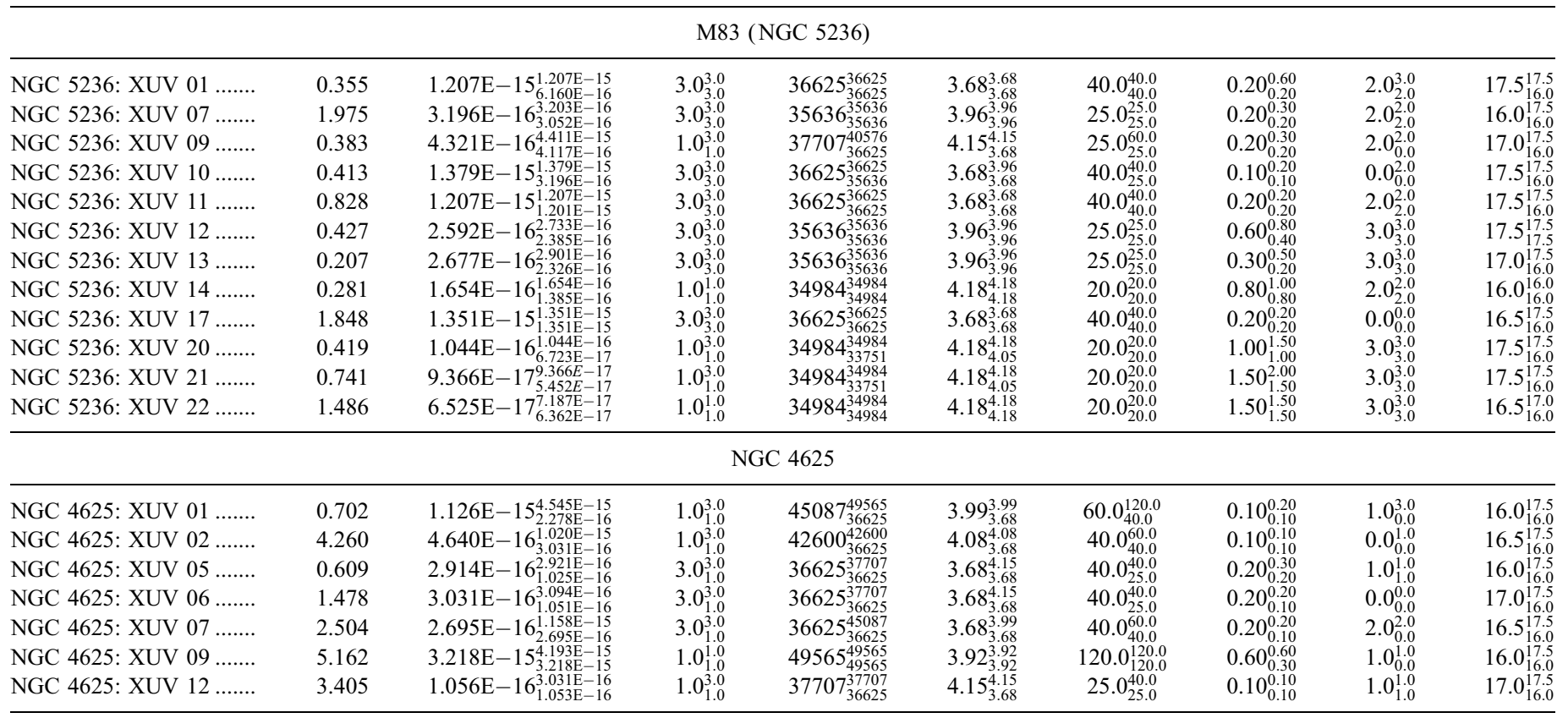

Notes.-Col. (1): Region identification. Col. (2): Best-fitting reduced $\chi^{2}$. Col. (3): H $\beta$ flux predicted by the photoionization models. Col. (4): Age of the star (Myr). Col. (5): Effective temperature (in K). Col. (6): Logarithm of the gravity. Col. (7): ZAMS mass ( $M_{\odot}$ ). Col. (8): Metal abundance of the gas and dust grains (in $Z_{\odot}$ ). Col. (9): Density of the gas (in atoms $\mathrm{cm}^{-3}$ ). Col. (10): Inner radius of the spherical gas distribution (in $\mathrm{cm}$ ). The $68.3 \%$ confidence intervals for each parameter considered separately are also given.

TABLE 5

Best-fitting Single-Star Photoionization Models (O/H-scaled N/O Ratio)

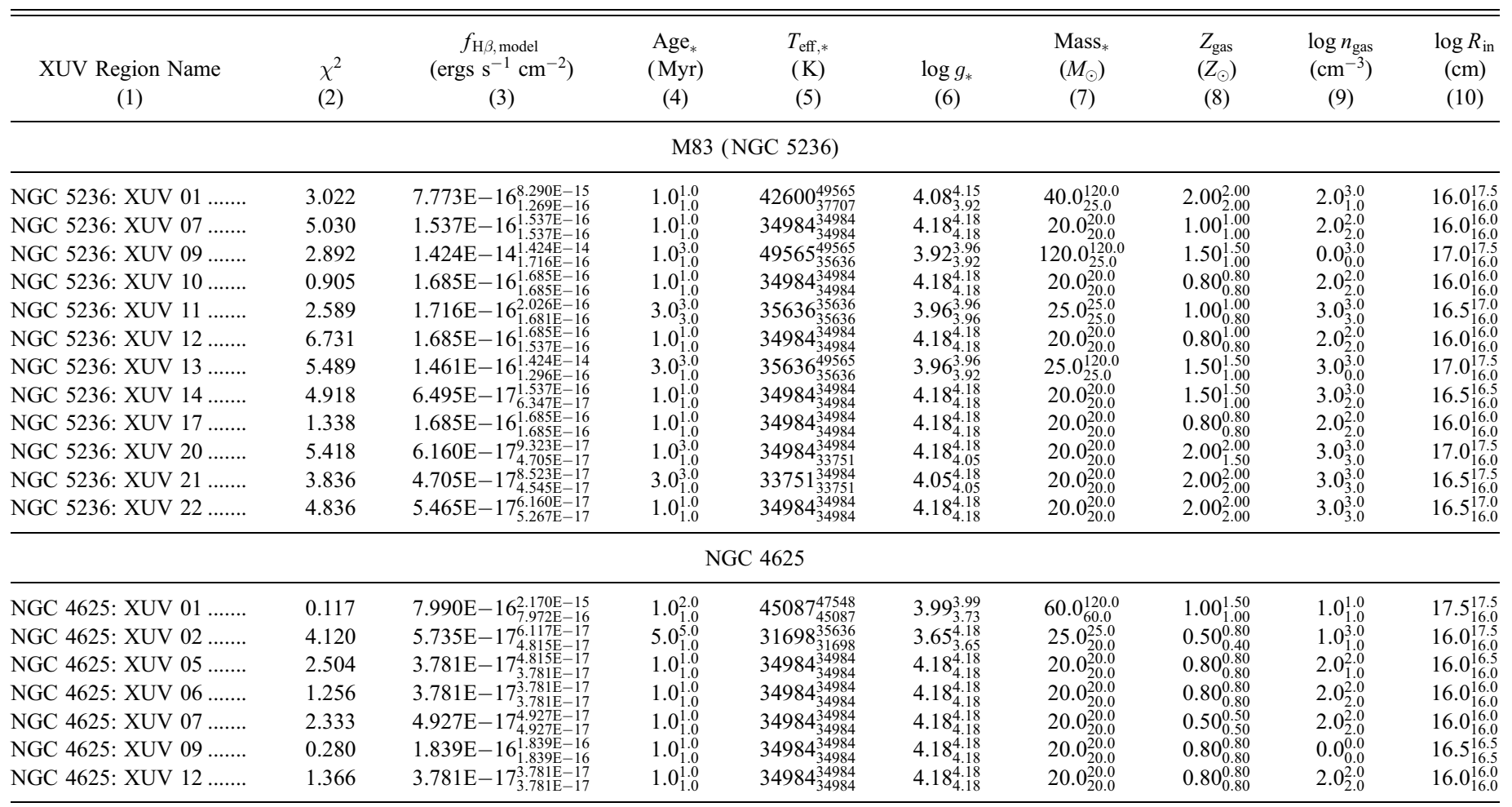

Notes.-Col. (1): Region identification. Col. (2): Best-fitting reduced $\chi^{2}$. Col. (3): H $\beta$ flux predicted by the photoionization models. Col. (4): Age of the star (Myr). Col. (5): Effective temperature (in K). Col. (6): Logarithm of the gravity. Col. (7): ZAMS mass ( $M_{\odot}$ ). Col. (8): Metal abundance of the gas and dust grains (in $Z \odot$ ). Col. (9): Density of the gas (in atoms $\mathrm{cm}^{-3}$ ). Col. (10): Inner radius of the spherical gas distribution (in $\mathrm{cm}$ ). The $68.3 \%$ confidence intervals for each parameter considered separately are also given. 
TABLE 6

Best-fitting Starburst Photoionization Models (Solar N/O Ratio)

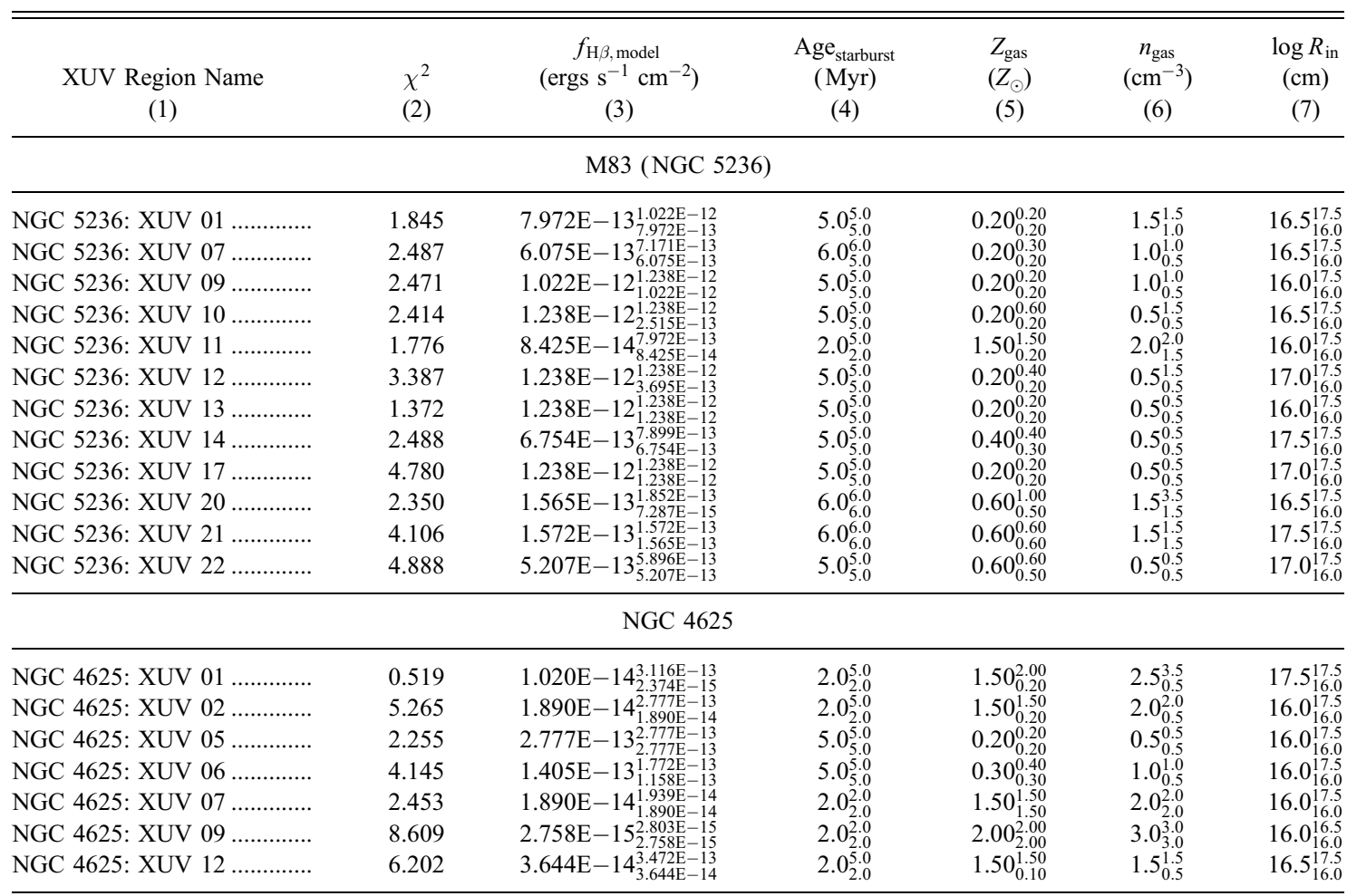

Notes.-Col. (1): Region identification. Col. (2): Best-fitting reduced $\chi^{2}$. Col. (3): H $\beta$ flux predicted by the photoionization models. Col. (4): Age of the starburst (Myr). Col. (5): Metal abundance of the gas and dust grains (in $Z_{\odot}$ ). Col. (6): Density of the gas (in $\mathrm{cm}^{-3}$ ). Col. (7): Inner radius of the spherical gas distribution (in $\mathrm{cm}$ ). A stellar mass of $10^{3} M_{\odot}$ was adopted for the ionizing starburst. The $68.3 \%$ confidence intervals for each parameter considered separately are also given (see text for more details).

TABLE 7

Best-fitting Starburst Photoionization Models (O/H-scaled N/O Ratio)

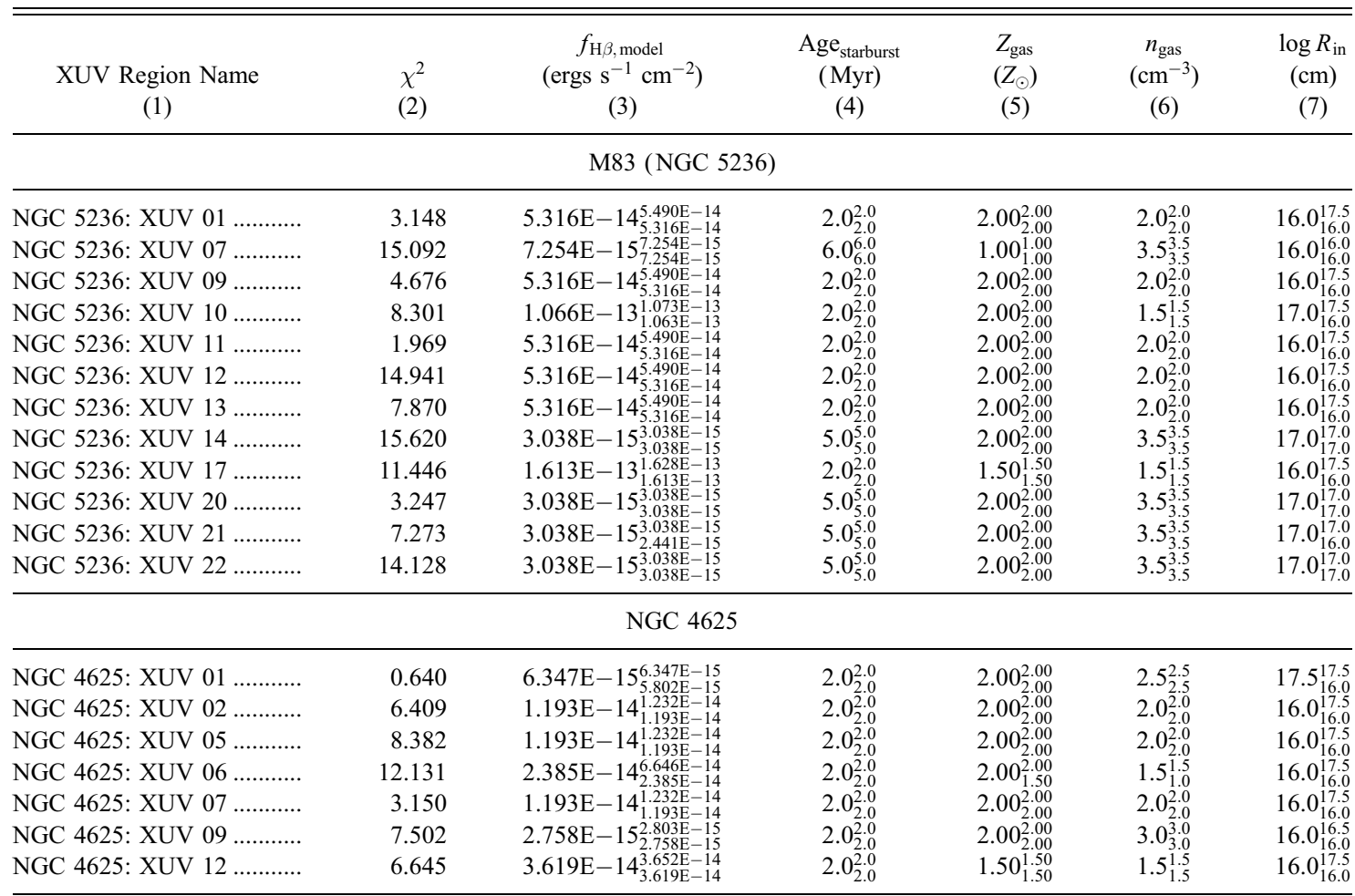

Notes. - Col. (1): Region identification. Col. (2): Best-fitting reduced $\chi^{2}$. Col. (3): H $\beta$ flux predicted by the photoionization models. Col. (4): Age of the starburst (Myr). Col. (5): Metal abundance of the gas and dust grains (in $Z_{\odot}$ ). Col. (6): Density of the gas (in $\mathrm{cm}^{-3}$ ). Col. (7): Inner radius of the spherical gas distribution (in $\mathrm{cm}$ ). A stellar mass of $10^{3} M_{\odot}$ was adopted for the ionizing starburst. The $68.3 \%$ confidence intervals for each parameter considered separately are also given (see text for more details). 
TABLE 8

Observed and Best-fitting Line Ratios

\begin{tabular}{|c|c|c|c|c|c|c|c|c|c|c|c|c|c|c|c|c|c|c|c|c|}
\hline \multirow[b]{2}{*}{$\begin{array}{l}\text { XUV Region Name } \\
\text { (1) }\end{array}$} & \multicolumn{4}{|c|}{ OBserved Line Ratios } & \multicolumn{4}{|c|}{ Single-StaR, Solar N/O Best Fit } & \multicolumn{4}{|c|}{ Single-StaR, O/H-scaled N/O Best Fit } & \multicolumn{4}{|c|}{ Starburst, Solar N/O Best Fit } & \multicolumn{4}{|c|}{ Starburst, O/H-SCALEd N/O Best Fit } \\
\hline & $\begin{array}{l}\mathrm{O} \text { II } / \mathrm{H} \beta \\
\quad(2)\end{array}$ & $\begin{array}{c}\mathrm{O} \text { III } / \mathrm{H} \beta \\
\quad(3)\end{array}$ & $\begin{array}{l}\mathrm{N}_{\text {II }} / \mathrm{H} \beta \\
\quad(4)\end{array}$ & $\begin{array}{l}\mathrm{S} \text { II } / \mathrm{H} \beta \\
\quad(5)\end{array}$ & $\begin{array}{l}\mathrm{O} \text { пा } / \mathrm{H} \beta \\
\quad(6)\end{array}$ & $\begin{array}{l}\mathrm{O} \text { III } / \mathrm{H} \beta \\
\quad(7)\end{array}$ & $\begin{array}{l}\mathrm{N} \text { II } / \mathrm{H} \beta \\
\quad(8)\end{array}$ & $\begin{array}{l}\mathrm{S} \text { II } / \mathrm{H} \beta \\
\quad(9)\end{array}$ & $\begin{array}{l}\mathrm{O} \text { II } / \mathrm{H} \beta \\
\quad(10)\end{array}$ & $\begin{array}{c}\mathrm{O} \underset{\text { III } / \mathrm{H} \beta}{ } \beta \\
\quad(11)\end{array}$ & $\begin{array}{c}\mathrm{N}_{\text {пा }} / \mathrm{H} \beta \\
\quad(12)\end{array}$ & $\begin{array}{l}\mathrm{S} \text { II } / \mathrm{H} \beta \\
\quad(13)\end{array}$ & $\begin{array}{l}\mathrm{O} \text { пा } / \mathrm{H} \beta \\
\quad(14)\end{array}$ & $\begin{array}{c}\mathrm{O} \text { III } / \mathrm{H} \beta \\
\quad(15)\end{array}$ & $\begin{array}{l}\mathrm{N} \text { II } / \mathrm{H} \beta \\
\quad(16)\end{array}$ & $\begin{array}{l}\mathrm{S} \text { пा } / \mathrm{H} \beta \\
\quad(17)\end{array}$ & $\begin{array}{l}\mathrm{O} \text { II } / \mathrm{H} \beta \\
\quad(18)\end{array}$ & $\begin{array}{l}\mathrm{O} \text { III } / \mathrm{H} \beta \\
\quad(19)\end{array}$ & $\begin{array}{c}\mathrm{N}_{\text {II }} / \mathrm{H} \beta \\
\quad(20)\end{array}$ & $\begin{array}{l}\mathrm{S} \text { пा } / \mathrm{H} \beta \\
\quad(21)\end{array}$ \\
\hline \multicolumn{21}{|c|}{ M83 (NGC 5236) } \\
\hline NGC 5236: XUV $01 \ldots . .$. & +0.110 & +0.490 & -0.434 & -0.654 & +0.116 & +0.410 & -0.484 & -0.509 & -0.172 & +0.476 & -0.623 & -0.209 & +0.122 & +0.362 & -0.521 & -1.100 & +0.116 & +0.397 & -0.738 & -0.968 \\
\hline NGC 5236: XUV $07 \ldots . .$. & +0.660 & -0.070 & -0.144 & -0.524 & +0.291 & +0.019 & -0.215 & -0.377 & +0.259 & -0.182 & -0.449 & -0.216 & +0.327 & -0.179 & -0.205 & -0.895 & +0.339 & -0.550 & -0.612 & -1.182 \\
\hline NGC 5236: XUV $09 . . .$. & +0.330 & +0.390 & -0.434 & -0.424 & +0.146 & +0.393 & -0.422 & -0.407 & +0.011 & +0.285 & -0.606 & -0.077 & +0.198 & +0.263 & -0.414 & -0.970 & +0.116 & +0.397 & -0.738 & -0.968 \\
\hline NGC 5236: XUV $10 \ldots . .$. & +0.310 & +0.030 & -0.354 & -0.304 & +0.216 & -0.067 & -0.408 & -0.338 & +0.364 & -0.028 & -0.520 & -0.182 & +0.240 & +0.176 & -0.348 & -0.844 & -0.041 & -0.027 & -0.739 & -1.013 \\
\hline NGC 5236: XUV $11 \ldots .$. & +0.310 & +0.480 & -0.564 & -0.654 & +0.116 & +0.410 & -0.484 & -0.509 & +0.043 & +0.277 & -0.709 & -0.456 & +0.204 & +0.387 & -0.751 & -0.954 & +0.116 & +0.397 & -0.738 & -0.968 \\
\hline NGC 5236: XUV $12 \ldots . .$. & +0.180 & +0.160 & -0.064 & -0.384 & +0.121 & +0.232 & -0.148 & -0.499 & +0.364 & -0.028 & -0.520 & -0.182 & +0.241 & +0.177 & -0.348 & -0.844 & +0.116 & +0.397 & -0.738 & -0.968 \\
\hline NGC 5236: XUV $13 \ldots . .$. & +0.280 & +0.320 & -0.234 & -0.554 & +0.209 & +0.249 & -0.202 & -0.566 & -0.138 & +0.201 & -0.590 & -0.494 & +0.241 & +0.178 & -0.348 & -0.843 & +0.116 & +0.397 & -0.738 & -0.968 \\
\hline NGC 5236: XUV $14 \ldots . .$. & +0.160 & -0.130 & -0.004 & -0.284 & +0.261 & -0.084 & +0.060 & -0.237 & +0.018 & -0.390 & -0.347 & -0.387 & +0.223 & -0.277 & -0.186 & -0.699 & +0.192 & -0.771 & -0.058 & -1.202 \\
\hline NGC 5236: XUV $17 \ldots . .$. & +0.630 & +0.050 & -0.394 & -0.194 & +0.307 & +0.031 & -0.230 & -0.156 & +0.364 & -0.028 & -0.520 & -0.182 & +0.241 & +0.177 & -0.348 & -0.844 & +0.147 & +0.077 & -0.865 & -0.918 \\
\hline NGC 5236: X & -0.050 & -0.540 & +0.076 & & +0.104 & -0.535 & +0.032 & & -0.201 & -0.478 & -0.309 & & +0.154 & -0.627 & -0.135 & & +0.192 & -0.771 & -0.058 & \\
\hline NGC 5236: XUV 21 ...... & -0.300 & -0.590 & +0.076 & -0.474 & -0.092 & -0.616 & -0.018 & -0.418 & -0.204 & -0.679 & -0.300 & -0.457 & +0.154 & -0.627 & -0.135 & -0.801 & +0.192 & -0.771 & -0.058 & -1.202 \\
\hline NGC 5236: XUV $22 \ldots . .$. & -0.290 & -0.270 & +0.076 & -0.323 & -0.074 & -0.426 & -0.028 & -0.440 & -0.194 & -0.456 & -0.310 & -0.468 & +0.102 & -0.362 & -0.213 & -0.700 & +0.192 & -0.771 & -0.058 & -1.202 \\
\hline \multicolumn{21}{|c|}{ NGC 4625} \\
\hline NGC 4625: XUV $01 \ldots .$. & +0.080 & +0.590 & -0.804 & & -0.096 & +0.506 & -0.787 & & +0.141 & +0.547 & -0.802 & & +0.240 & +0.654 & -0.806 & & +0.197 & +0.690 & -0.748 & \\
\hline NGC 4625: XUV $02 \ldots . .$. & +0.730 & +0.300 & -0.594 & -0.484 & +0.128 & +0.271 & -0.522 & -0.372 & +0.386 & +0.333 & -0.709 & +0.141 & +0.204 & +0.387 & -0.751 & -0.954 & +0.116 & +0.397 & -0.738 & -0.968 \\
\hline NGC 4625: XUV $05 \ldots . .$. & +0.470 & +0.220 & -0.354 & -0.333 & +0.240 & +0.239 & -0.336 & -0.312 & +0.364 & -0.028 & -0.520 & -0.182 & +0.241 & +0.178 & -0.348 & -0.843 & +0.116 & +0.397 & -0.738 & -0.968 \\
\hline NGC 4625: XUV $06 \ldots . .$. & +0.580 & -0.070 & -0.354 & -0.203 & +0.307 & +0.031 & -0.230 & -0.156 & +0.364 & -0.028 & -0.520 & -0.182 & +0.277 & -0.146 & -0.217 & -0.838 & -0.041 & -0.027 & -0.739 & -1.013 \\
\hline NGC 4625: XUV $07 \ldots .$. & +0.530 & +0.440 & -0.634 & -0.583 & +0.119 & +0.406 & -0.483 & -0.510 & +0.361 & +0.528 & -0.754 & -0.100 & +0.204 & +0.387 & -0.751 & -0.954 & +0.116 & +0.397 & -0.738 & -0.968 \\
\hline NGC 4625: XUV 09 ..... & +0.710 & +0.960 & -0.394 & & +0.162 & +0.814 & -0.385 & 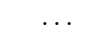 & +0.722 & +0.888 & -0.337 & $\ldots$ & +0.223 & +0.918 & -0.783 & & +0.221 & +0.917 & -0.735 & \\
\hline NGC 4625: XUV $12 \ldots .$. & +0.620 & +0.070 & -0.644 & -0.234 & +0.157 & +0.116 & -0.468 & -0.410 & +0.364 & -0.028 & -0.520 & -0.182 & +0.105 & +0.038 & -0.690 & -0.947 & +0.147 & +0.077 & -0.865 & -0.918 \\
\hline
\end{tabular}

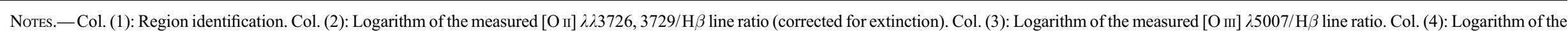

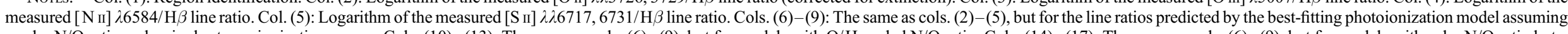

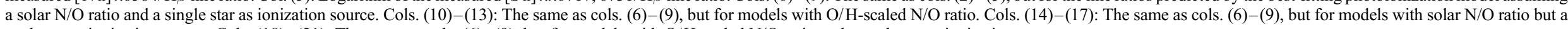
starburst as ionization source. Cols. (18)-(21): The same as cols. (6)-(9), but for models with $\mathrm{O} / \mathrm{H}$-scaled N/O ratio and a starburst as ionization source. 
of the regions, which indicates that these single-star photoionization models provide a good fit to the line ratios measured. The fact that they are not much smaller than 1 also indicates that we are not overfitting our data. The high reduced $\chi^{2}$ found in some of the regions might be due to these regions being powered by a few stars of different effective temperature or by a larger composite stellar population such as a stellar cluster (see below). We have estimated the uncertainties in the properties derived by determining the minimum (subscripts in Tables 4 and 5) and maximum (superscripts in Tables 4 and 5) values of each parameter that result in values of the absolute (i.e., not reduced) $\chi^{2}$ that differ from the minimum $\chi^{2}$ by less than $\Delta \chi^{2}=1$ (see Avni 1976). This yields the $68.3 \%$ confidence intervals in each parameter when they are considered separately. Table 4 shows that while the ages and masses of the ionizing stars and the metal abundance of the gas are relatively well constrained, the inner radius of the gas distribution is very poorly constrained by our measurements.

In roughly half of the regions (NGC 5236: XUV 01, 07, 10, 11, and 17 and NGC 4625: XUV 01, 05, and 09) the $\mathrm{H} \beta$ fluxes predicted by the best-fitting model are comparable to or a factor of a few above (explicable by slit aperture effects) the fluxes measured through the slits (Table 2). There are some cases (NGC 5236: XUV 09, 12, and 14 and NGC 4625: XUV 02, 06, and 07) where the measurements are above the models but only by a factor of $\sim 2-5$. These $\mathrm{H}$ II regions could well be powered by a very few massive stars. Finally, in a few regions (NGC 5236: XUV 13, 20, 21, and 22 and NGC 4625: XUV 06 and 12) the slit $\mathrm{H} \beta$ fluxes are more than a factor of 10 above those predicted by the models, suggesting that the source of the ionizing radiation is a relatively massive stellar cluster. This was expected in the case of regions NGC 5236: XUV 20, 21, and 22 since they are very luminous regions in the optical disk of M83.

In the case of M83 the ZAMS masses derived for the ionizing stars of the XUV regions are in the range $20-40 M_{\odot}$ with a bestfitting age of 1-3 Myr. With respect to the properties of the gas, the densities and inner radii show a wide range of values between 1 and $10^{3} \mathrm{~cm}^{-3}$ and between $10^{16}$ and $10^{17.5} \mathrm{~cm}$, respectively. The best-fitting single-star models for NGC 4625 yield lower gas densities and somewhat higher temperatures and masses for the ionizing stars than those of M83.

The gas metal abundances nicely agree with those derived in $\S 3.2$ using the R23 ratio. Indeed, while M83 shows a strong metallicity gradient with abundances between $Z_{\odot} / 10$ and $Z_{\odot} / 4$ in the outer disk and close or even above solar in the optical disk, the XUV disk regions of NGC 4625 (except for NGC 4625: XUV 09, with $\chi^{2}>5$ ) show best-fitting metallicities that are either $Z_{\odot} / 5$ or $Z_{\odot} / 10$.

In light of these results, we can now confidently say that metal abundances as low as $12+\log (\mathrm{O} / \mathrm{H})=7.86$ (regions NGC 5236: XUV 01 and NGC 5236: XUV 10) and $12+\log (\mathrm{O} / \mathrm{H})=7.94$ (NGC 4625: XUV 01) are found in the XUV disks of M83 and NGC 4625, respectively. These metallicities are comparable to the lowest oxygen abundances found by van Zee et al. (1998b) $[12+\log (\mathrm{O} / \mathrm{H})=7.92$ in NGC 5457] and Ferguson et al. $(1998)[12+\log (\mathrm{O} / \mathrm{H})=7.95$ in NGC 1058] in the outermost regions of a sample of spiral galaxies. Note that in the case of Ferguson et al. (1998) only galaxies in Ferguson (1997) that showed very extended $\mathrm{H}$ I and extreme outer disk star formation were analyzed.

\subsubsection{Best-fitting Starburst Models}

Table 6 shows the properties and reduced $\chi^{2}$ of the best-fitting starburst models with solar $\mathrm{N} / \mathrm{O}$ ratio. The reduced $\chi^{2}$ values are larger than (i.e., worse than) or, at best, comparable to those obtained from the corresponding single-star photoionization model. This indicates that for low stellar masses such as those of the XUV complexes (Gil de Paz et al. 2005) the shape of the ionizing spectrum is different from that of a modeled composite stellar population, which are usually built for massive star-forming regions and starburst galaxies. In particular, even in those cases where the presence of more than one ionizing star is suspected, stochastic effects on the upper IMF might result in the ionizing radiation from these regions being dominated by only two or three of these stars, which would lead to an ionizing spectrum different from that of a low-mass stellar cluster with a continuous IMF, such as those generated by the Starburst99 population synthesis models. Unfortunately, the constraints imposed by the few line ratios measured in this study are limited, so the determination of the number and masses of the ionizing stars present in each of these regions is beyond our reach. High-resolution imaging observations using the Hubble Space Telescope would partially solve this problem by resolving individual massive stars at the distance of the nearest XUV disks. As expected, younger best-fitting starburst models typically correspond to regions where the effective temperature of the best-fitting singlestar model is higher. The $68.3 \%$ confidence intervals in metal abundance of some of the regions in NGC 4625 are noticeably wider than those found using single-star models. Again, we find that the inner radius of the dust distribution is very poorly constrained.

We note that although for the low metal abundances of the regions analyzed here the differences between the CoStar library and Pauldrach/Hillier stellar atmospheres used in Starburst99 are not as dramatic as for $Z \geq Z_{\odot}$ (Smith et al. 2002), differences between the two models could also partially explain the different best-fitting $\chi^{2}$ values achieved. Should that be the case, the massive stars present in the emission-line regions in the XUV disk would likely have a harder ionizing spectrum (as in the CoStar library; see Smith et al. 2002) than the O stars included in the Starburst99 models.

However, we should emphasize here that the fact that singlestar models better reproduce the line ratios was suspected already based uniquely on the low emission-line fluxes measured in these regions (see Table 2). Unfortunately, as we commented in $\S 3.1$, some of these spectroscopic fluxes might be significantly affected by aperture effects, so our results could not be based solely on that fact.

\subsection{Metallicity Gradients}

The oxygen metal abundances of the $\mathrm{H}$ II regions in the disk of M83 are found to decrease with the galactocentric distance (Fig. 7a). If we assume that for regions NGC 5236: XUV 09 to NGC 5236: XUV 14 the low-metallicity branch of the R23 calibration is the most appropriate, the best linear fit yields a metallicity gradient of $d[\mathrm{O} / \mathrm{H}]=-0.112$ and $-0.069 \mathrm{dex} \mathrm{kpc}^{-1}$ using the M91 and PT05 methods, respectively, with

$$
\begin{array}{ll}
12+\log (\mathrm{O} / \mathrm{H})=-0.112 r(\mathrm{kpc})+9.36 ; & \sigma=0.37 \mathrm{dex} \\
12+\log (\mathrm{O} / \mathrm{H})=-0.069 r(\mathrm{kpc})+8.60 ; & \sigma=0.34 \mathrm{dex}
\end{array}
$$

respectively. Here we have combined our metallicity measurements for the XUV disk with those derived from the line ratios of $\mathrm{H}$ II regions in the optical disk (Webster \& Smith 1983) using the same methodology as for the XUV disk abundances (open circles in Fig. 7a). If the high-metallicity branch of the R23 calibration 

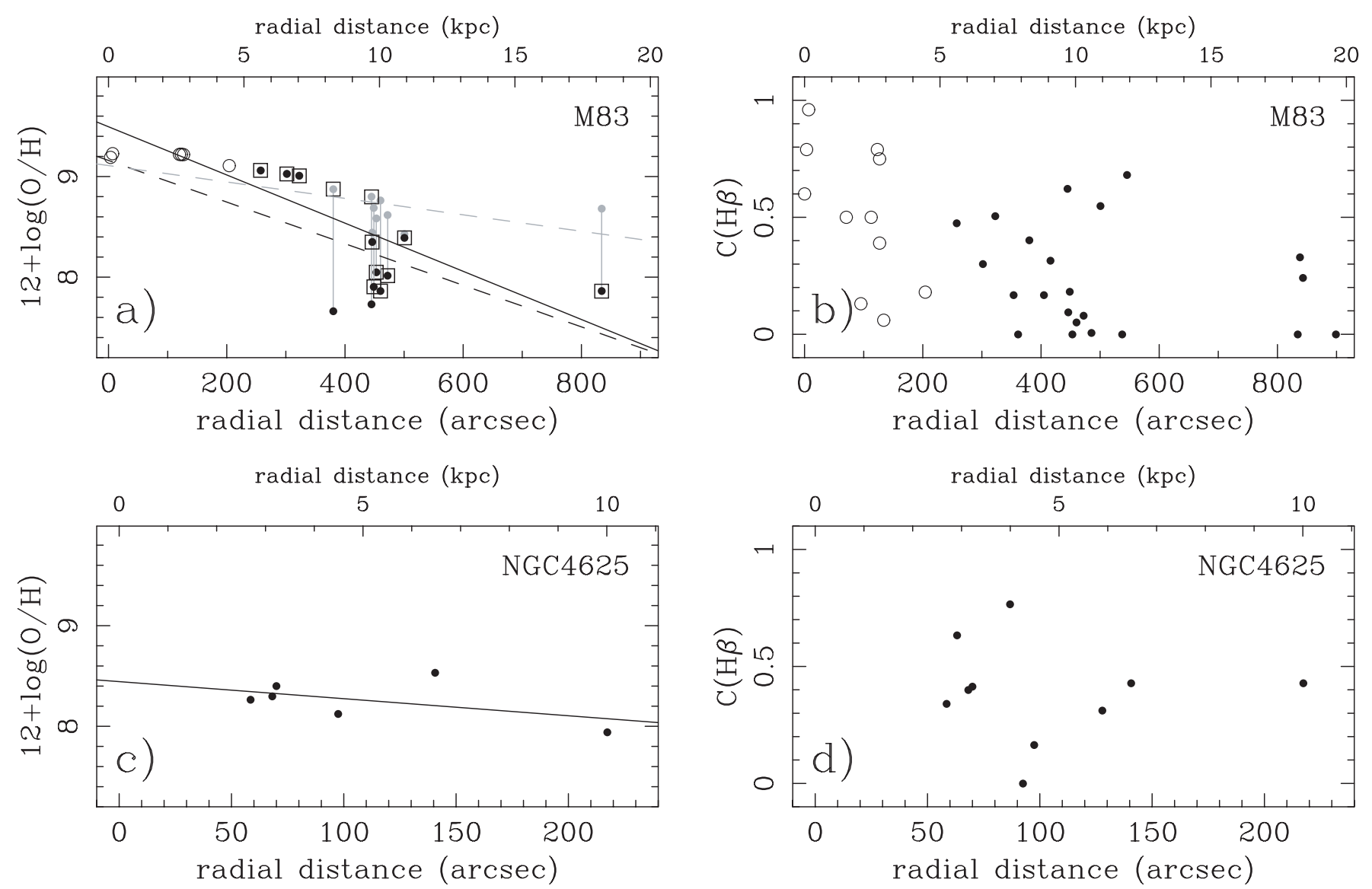

FIG. 7.- (a) Radial distribution of the nebular oxygen abundance in M83. Filled circles correspond to metallicities derived from the line ratios measured in this work. Open circles are the metallicities derived using the line ratios measured by Webster \& Smith (1983) in six H II regions in the inner optical disk of M83. The black and gray circles are the metallicities obtained assuming the low- and high-metallicity branch, respectively, for the calibration of the R23 parameter for regions NGC 5236: XUV 09-14. The black and gray dashed lines are the corresponding best linear fits. The solid line is the best fit obtained after using the metal abundance information provided by the best-fitting single-star photoionization models (open squares; see text for details). These oxygen abundances were derived using the M91 calibration of the R23 parameter. Coefficients for all these fits and those obtained using the calibration of PT05 are given in the text. (b) Radial distribution of the internal extinction in $\mathrm{H} \beta[C(\mathrm{H} \beta)]$ for M83. We have adopted the Galactic color excesses in the direction of M83 and NGC 4625 to be 0.066 and 0.018 mag, respectively (Schlegel et al. 1998). Symbols have the same meaning as in panel $(a)$. $(c, d)$ Same as $(a, b)$, but for NGC 4625.

is now assumed for regions NGC 5236: XUV 09 to NGC 5236: XUV 14, the metallicity gradient is much shallower,

$12+\log (\mathrm{O} / \mathrm{H})=-0.051 r(\mathrm{kpc})+9.26 ; \quad \sigma=0.16 \mathrm{dex}$,

$12+\log (\mathrm{O} / \mathrm{H})=-0.017 r(\mathrm{kpc})+8.51 ; \quad \sigma=0.25 \mathrm{dex}$,

for M91 and PT05, respectively. Finally, if the branch of the R23 calibration is assigned based on the metal abundance of the bestfitting Cloudy single-star photoionization model with solar N/O, i.e., low-metallicity branch for all regions except for NGC 5236: XUV 12, 14, 20, 21, and 22, the result is

$$
\begin{array}{ll}
12+\log (\mathrm{O} / \mathrm{H})=-0.100 r(\mathrm{kpc})+9.41 ; & \sigma=0.30 \mathrm{dex}, \\
12+\log (\mathrm{O} / \mathrm{H})=-0.058 r(\mathrm{kpc})+8.64 ; & \sigma=0.30 \mathrm{dex}
\end{array}
$$

for M91 and PT05, respectively. These values are slightly steeper and shallower, respectively, than the metallicity gradient

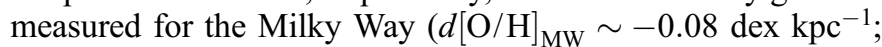
Boissier \& Prantzos 1999 and references therein) and those derived for a sample of spiral galaxies with oxygen abundance measurements at large radii (van Zee et al. 1998b; Ferguson et al. 1998). If we now express the metallicity gradient in units of the blue-band scale length $\left(r_{d}\right)$ or D25 radius $\left(r_{25}\right)$, we obtain $-0.198 \mathrm{dex} / r_{d}$ and $-1.01 \mathrm{dex} / r_{25}$, respectively, in the case of the M91 calibration and $-0.115 \mathrm{dex} / r_{d}$ and $-0.59 \mathrm{dex} / r_{25}$ with the PT05 calibration. These values are similar to the "universal" gradient of $-0.2 \mathrm{dex} / r_{d}$ (or $-0.8 \mathrm{dex} / r_{25}$ ) proposed by Prantzos $\&$ Boissier (2000; see also Garnett et al. 1997) for nonbarred disk galaxies and within the range of values found by van Zee et al. (1998b) and Ferguson et al. (1998) in spirals with outer disk star formation.

In light of Figure $7 a$, instead of a single linear fit, the radial dependence of metal abundance seems to suggest a smooth metallicity gradient below $10 \mathrm{kpc}$ and sharp decrease in metallicity from $\sim Z_{\odot}$ to $Z_{\odot} / 5$ (if the M91 calibration is used) at that radius. This behavior could be explained if there exists a cutoff radius beyond which the disk of M83 is significantly less efficient in forming stars and it is allowed to evolve mostly viscously (Clarke 1989). Note, however, that this might also be the consequence of the large uncertainties expected for any R23-based metallicity estimate at abundances close to half the solar value. A larger number of metallicity measurements, especially in the range $10-15 \mathrm{kpc}$, and more precise estimates of the metallicity using the $[\mathrm{O}$ III] $\lambda 4363$ temperature-sensitive line would be required to confirm (or rule out) this. 
In the case of NGC 4625, where the low-metallicity branch can be safely assumed, the best linear fits obtained are

$$
\begin{array}{ll}
12+\log (\mathrm{O} / \mathrm{H})=-0.037 r(\mathrm{kpc})+8.44 ; & \sigma=0.20 \mathrm{dex}, \\
12+\log (\mathrm{O} / \mathrm{H})=-0.017 r(\mathrm{kpc})+8.16 ; & \sigma=0.39 \mathrm{dex},
\end{array}
$$

for M91 and PT05, respectively. The metallicity gradient derived, $\Delta[\mathrm{O} / \mathrm{H}] / \Delta R=-0.037 \pm 0.032 \mathrm{dex} \mathrm{kpc}^{-1}$ (using the M91 calibration), is very modest. Even after normalizing this value by the galaxy scale length or D25 radius, this is one of the shallowest gradients measured in disk galaxies, $-0.080 \mathrm{dex} / r_{25}$. Note, however, that NGC 4625 is significantly less luminous $\left(M_{B}=\right.$ $-17.4 \mathrm{mag}$ ) than the spiral galaxies where metallicity gradients have been traditionally measured.

The dust attenuation also seems to decrease with the galactocentric distance in the case of M83 although with a very large dispersion (Fig. $7 b$ ). The fact that this scatter is present even when neighboring regions are considered (e.g., NGC 5236: XUV 07 and NGC 5236: XUV 08) suggests a patchy dust distribution. In the case of NGC 4625 (see Fig. 7d) no obvious trend with the galactocentric distance is found.

It is also remarkable that positive and relatively large values of $C(\mathrm{H} \beta)$ are measured very far out into the disks, showing the presence of dust in these galaxies at distances larger than $18 \mathrm{kpc}$ in the case of M83 and more than $10 \mathrm{kpc}$ in NGC 4625 (for an example of dust emission associated with an extended $\mathrm{H}$ I disk see Popescu \& Tuffs 2003).

\section{DISCUSSION}

\subsection{Nature of the XUV Disks: XUV Complexes}

From the analysis carried out in this paper and from previous studies on the XUV disk phenomenon (Thilker et al. 2005a; Gil de Paz et al. 2005) it is now clear that the XUV emission is due to young stars associated with low-mass stellar associations located at large galactocentric distances. The line ratios measured in those XUV complexes showing line emission indicate the presence of UV ionizing radiation being emitted locally in these regions. It is therefore unlikely that the nonionizing UV light responsible for the XUV emission seen by GALEX is due to scattering by dust, as in the case of the polar regions of the starburst galaxy M82 (Hoopes et al. 2005). Our measurements also exclude planetary nebulae (with very high temperatures and highly excited gas; see $\S 3.1$ ) as being the agents responsible for the observed XUV emission. Finally, a significant contribution by blue horizontal branch (HB) stars is highly unlikely considering (1) the blue NUV-optical colors of the XUV disks, (2) the high equivalent widths measured in some XUV complexes, and (3) the morphology (resembling spiral arms) of the XUV emission, all unusual circumstances in objects with UV emission dominated by evolved stars (globular clusters, elliptical galaxies) but common in galaxies forming massive stars in recent epochs.

Our results also support the conclusions of Gil de Paz et al. (2005) that suggested that the majority of the admittedly scarce emission-line sources found in the XUV disks of these galaxies seem to be powered by single stars, with masses between 20 and $40 M_{\odot}$ in most cases. As a consequence of this, a stochastic treatment of the IMF (e.g., Cerviño 1998) is necessary in order to properly analyze the properties of the stellar populations in these XUV disks and perhaps in the outer edges of all spiral galaxies. In particular, knowledge on the fraction of low-mass stellar complexes that, once stochastic effects are properly accounted for, would form at least one ionizing star is key to understanding the observed differences between the UV and $\mathrm{H} \alpha$ light profiles of XUV disks (see, e.g., Meurer et al. 2004).

In this sense, it has been recently argued (Boissier et al. 2007) that the lack of $\mathrm{H} \alpha$ emission in the azimuthally averaged profiles of spiral galaxies beyond a given radius, which is commonly associated with the existence of an intrinsic star formation threshold (e.g., Martin \& Kennicutt 2001), could instead be a consequence of the fact that at those low levels of star formation the number of ionizing stars expected to be found (at a given time) inside one of these annuli of the radial profile is less than unity. Under these circumstances, the chances of catching one of these ionizing stars are very low, leading to an effective lack of $\mathrm{H}$ II regions in most cases, but not necessarily to a lack of time-integrated star formation.

\subsection{Nature of the XUV Disks: Extended $\mathrm{H} \mathrm{I}$, Cause or Consequence?}

Another property of the XUV disks is that they are always found in galaxies with very extended $\mathrm{H}$ I distributions (see Tilanus \& Allen [1993] in the case of M83 and Bush \& Wilcots [2004] for NGC 4625). This is not surprising since gas is of course a necessary ingredient for the formation of the stars responsible for the UV emission and the neutral-to-molecular gas ratio is thought to increase with the galactocentric radius in field spiral galaxies (e.g., Kohta et al. 2001). However, the fact that significant amounts of $\mathrm{H} \mathrm{I}$ (compared with their stellar content) are still found at the positions of these UV complexes suggests that the efficiency in converting neutral gas into stars is relatively low and that, under the right circumstances, these XUV disks could maintain the observed level of star formation for a cosmologically significant period of time. The star formation rate per unit area of these XUV disks can be obtained from the azimuthally averaged FUV surface brightness after a correction for extinction of $A_{\mathrm{FUV}}=1 \mathrm{mag}$ is adopted. ${ }^{14}$ If we now use the gas surface density profile of Thilker et al. (2005a), the consumption timescale derived for M83 is $\geq 6 \mathrm{Gyr}$ at all radii, only considering the $\mathrm{H}$ I. In the case of NGC 4625 the consumption timescale is $\sim 3 \mathrm{Gyr}$ in the innermost parts of the XUV disk and rises up to values of the order of a Hubble time in the outer regions (Gil de Paz et al. 2005).

Alternatively, it could be argued that the presence of an extended $\mathrm{H}$ I disk is a consequence of the XUV emission instead of a necessary preexisting condition (Allen 2002). Under this scenario, the extended $\mathrm{H}$ I emission would be the result of the dissociation of $\mathrm{H}_{2}$ in photodissociation regions (PDRs) by the UV photons emitted by the same newly formed stars responsible for the XUV emission.

However, in most of these XUV disks the complexes responsible for the FUV emission cover only a fraction of the area of the $\mathrm{H}$ I disk. Therefore, in order for this scenario to be valid in the case of the XUV disks, the time for the reformation of the $\mathrm{H}_{2}$ molecule on the surface of dust grains from atomic hydrogen, $\tau_{\text {form }}$, should be larger than, or at least comparable to, the average time a particular region in the disk is embedded in FUV radiation. This condition can be written as

$$
\tau_{\text {form }} \gtrsim \frac{A_{\mathrm{H}}}{A_{\mathrm{FUV}}} \tau_{\mathrm{FUV}},
$$

\footnotetext{
14 This corresponds to an average observed (FUV - NUV) color of $0.3 \mathrm{mag}$ and the relationship between (FUV - NUV) color and $A_{\mathrm{FUV}}$ given by Boissier et al. (2007). Note that $\mathrm{UV}$ magnitudes are in $\mathrm{AB}$ scale and optical magnitudes are referred to Vega throughout the text.
} 
where $A_{\mathrm{H}}$ is the area of the XUV disk where $\mathrm{H}$ I emission is detected, $A_{\mathrm{FUV}}$ is the area of the disk reached by the FUV photons (responsible for the $\mathrm{H}_{2}$ photodissociation in PDRs), and $\tau_{\mathrm{FUV}}$ is the timescale of the FUV emission, which is of the order of a few hundred Myr for an instantaneous burst.

The $\mathrm{H}_{2}$ reformation rate can be obtained using the expression given by Hollenbach \& McKee (1979),

$$
R_{\mathrm{H}_{2}} \equiv \frac{d n_{\mathrm{H}_{2}}}{d t}=\frac{1}{2} n_{\mathrm{H}} v_{\mathrm{H}} \sigma \xi \eta n_{g},
$$

where $n_{\mathrm{H}}$ is the number density of hydrogen atoms (in atomic form), $v_{\mathrm{H}}$ is their thermal speed, $\sigma$ is the cross section of the grain, $\xi$ is the sticking coefficient, $\eta$ is the probability of bond formation once two hydrogen atoms encounter each other, and $n_{g}$ is the number density of dust grains in the ISM. Here we assume that the contribution of the $\mathrm{H}^{-}$process to the formation of $\mathrm{H}_{2}$ is negligible. From the expression of the reformation rate given above, the reformation time, defined as the time necessary for half of the total hydrogen atoms to be in molecular form, can be written as

$$
\tau_{\text {form }}=\frac{\ln 2}{v_{\mathrm{H}} \sigma \xi \eta n_{g}} .
$$

For simplicity, we assume a gas temperature of $70 \mathrm{~K}$ (Hughes et al. 1971), which yields a thermal speed for the hydrogen atoms of $1.2 \times 10^{5} \mathrm{~cm} \mathrm{~s}^{-1}$ (Spitzer 1978). The cross section of the grain is computed as $\pi a^{2}$, where $a$ is the typical radius of the grains in the ISM $(0.1 \mu \mathrm{m}$; Jones \& Merrill 1976). The sticking coefficient is adopted to be $\xi=0.3$ and $\eta=1$ (Vidali et al. 2005; Hollenbach $\&$ Salpeter 1971). The number density of grains is estimated to be $2 \times 10^{-12} n_{\mathrm{H}}$ after an average grain material density of $2 \mathrm{~g} \mathrm{~cm}^{-3}$ (Draine \& Lee 1984) and the Galactic ISM gas-to-dust mass ratio are adopted $\left(R_{g / d} \sim 100 ;\right.$ Knapp \& Kerr 1974). This yields a reformation time of

$$
\tau_{\text {form }}(\mathrm{Myr}) \approx \frac{10^{3}}{n_{\mathrm{H}}},
$$

where $n_{\mathrm{H}}$ is expressed in $\mathrm{cm}^{-3}$. Allen (2002) gives a similar expression although his reformation timescales are somewhat smaller than those given by equation (12). This is in part due to the fact that he uses parameters typical of PDRs, while in our case the reformation of the $\mathrm{H}_{2}$ is expected to take place in a more rarefied ISM.

For XUV disks where the FUV emission covers a high fraction of the disk (like in the case of the inner XUV disk of NGC 4625; Gil de Paz et al. 2005) the reformation time might fulfill the condition imposed by equation (9) as long as the gas is not very dense. On the other hand, in M83, where the FUV emission covers only $\sim 10 \%$ of the extended $\mathrm{H}$ I disk, the gas density required for the hydrogen to stay in atomic form is very low $\left(n_{\mathrm{H}}<1 \mathrm{~cm}^{-3}\right)$. We note that the Galactic gas-to-dust ratio assumed above corresponds to an ISM with a higher abundance of metals and probably dust grains than that expected to be present in the XUV disk of M83, where a larger $R_{g / d}$ might exist (see Galliano et al. 2003, 2005). Moreover, the size of the PDRs might be somewhat larger than that of the FUV-bright regions seen in the GALEX images, which would lead to a smaller value of $A_{\mathrm{H}} / A_{\mathrm{FUV}}$.

Therefore, we cannot definitively exclude the possibility that a significant fraction of the extended $\mathrm{H}$ I associated with the XUV disks is a consequence of the photodissociation of $\mathrm{H}_{2}$ in PDRs.

A more detailed analysis of the relation between gas and recent star formation in XUV disks, including a study of the star formation threshold and the star formation law, will be the subject of a future paper.

\subsection{Past and Future Evolution of the XUV Disks}

The very low surface brightnesses of these XUV disks at optical wavelengths and, consequently, the very blue UV-optical colors of their stellar populations led to the suggestion that these might be the first generations of stars being formed in these regions (Madore et al. 2004; Gil de Paz et al. 2005). However, the oxygen abundances derived for the emission-line regions of M83 and NGC $4625\left(Z \sim Z_{\odot} / 5-Z_{\odot} / 10\right)$ indicate that in spite of being relatively unevolved chemically these are not the first stars to have formed in the outermost regions of these galaxies. Some star formation activity must have taken place in these regions in the past in order to enrich the ISM to its present levels.

There are many ways to produce such an enrichment of the ISM:

1. Low-level continuous star formation.-If the star formation history of the XUV disks has been similar to that of young spiral disks during their early formation at high redshift, this level of chemical enrichment could have been reached in a more or less continuous way only $\sim 1$ Gyr after the first stars formed (see, e.g., Boissier \& Prantzos 2000). A scenario where the first stars formed much earlier than 1 billion years ago and have maintained the same level of star formation is unlikely since that would result in redder colors than those measured in the XUV disks. In particular, if we correct the color profiles of NGC 4625 given in Gil de Paz et al. (2005) for extinction using the relation between the (FUV - NUV) color and $A_{\mathrm{FUV}}$ found by Boissier et al. (2007) for the disks of spiral galaxies (assuming the Galactic extinction law), the corrected (NUV $-B$ ) color would range between 0.4 mag (inner XUV disk) and -0.5 mag (outer XUV disk). For this range of colors any stellar population with an approximately constant star formation rate (and $Z=Z_{\odot} / 5$ ) would necessarily be younger than 1 Gyr (e.g., Bruzual \& Charlot 2003). On the other hand, the corrected $(B-R)$ color is approximately flat with a value of $\sim 0.6-0.7 \mathrm{mag}$. This value is consistent with a 2-5 Gyr old continuously forming stellar population or with a 500 Myr old instantaneous burst. However, since the photometry errors in $(B-R)$ are large compared with its age sensitivity, significantly younger (or older) ages are also possible.

As noted above, the amount of gas in the XUV disks of these systems is large enough to maintain the current level of star formation for several Gyr and up to a Hubble time depending on the XUV disk and the region of the XUV disks considered, especially once the contribution of a possible molecular component to the total gas mass is included. It is therefore conceivable that these galaxies can keep forming stars in their outermost regions at the current rate for a few Gyr and that the optical disk will steadily grow thanks to the accumulation of the light from the resulting long-lived low- and intermediate-mass stars.

2. Episodic star formation.-Alternatively, an episodic star formation with brief epochs of active star formation followed by long quiescent periods could also have led to this level of chemical enrichment. If this were the case, other (now quiescent) galaxies might have gone through one or several of these episodes during their lives. Thilker et al. (2005b) have recently proposed that the so-called antitruncated disks identified by Erwin et al. (2005) in early-type spirals could be the evolved counterparts of the massive XUV disk population. The acquisition of deep groundbased multiwavelength surface photometry and single-star colormagnitude diagrams would greatly benefit the determination of the star formation history of XUV disks along with its possible 
connection with the antitruncated disks. Deeper optical spectroscopy of some of these regions with the aim of determining more precise $\mathrm{O} / \mathrm{H}$ and N/O measurements should also provide additional clues on the star formation histories of XUV disks. Some of these efforts are currently underway.

\section{CONCLUSIONS}

Analysis of the emission-line spectra of a sample of $\mathrm{H} \alpha$ selected regions in the XUV disks of M83 and NGC 4625 allows us to draw the following conclusions regarding the properties of these disks:

1. The line ratios measured in the emission-line regions of the XUV disks of M83 and NGC 4625 show that their ionizing radiation is emitted by young stars and is due to neither scattering by dust nor hot evolved stars, such as blue HB or post-AGB stars.

2. The metal abundances derived, both from the R23 parameter and by comparison of multiple line ratios with the predictions of photoionization models, are nonprimordial with oxygen abundances between $Z_{\odot} / 10$ and $Z_{\odot} / 4$ in NGC 4625 . The oxygen abundance in M83 increases from $\sim Z_{\odot} / 10$ in the outermost regions of the XUV disk to close to solar (or even supersolar if the M91 calibration is adopted) abundances in the optical disk. The same behavior is observed for the radial distribution of the dust extinction: it is approximately flat in the case of NGC 4625 and shows a progressive decrease with increasing galactocentric distance (although with a large scatter) in M83. The comparison of the observed line ratios with the model predictions favors a high $\mathrm{N} / \mathrm{O}$ abundance ratio for the XUV disk of M83. This could be due to the infall of pristine gas in regions of secondary nitrogen production or be the natural outcome of a top-light IMF.

3. The emission-line luminosities and ratios measured indicate that most of the $\mathrm{H}$ in regions analyzed are photoionized by single massive stars and only a few are powered by a stellar cluster. This fact, along with the low azimuthally averaged star formation rates derived, suggests that stochastic effects both on the upper IMF (Cerviño 1998) and on the notion of star formation threshold (Boissier et al. 2007) should not be ignored when studying the properties of the outer edges of spiral galaxies in general and of XUV disks in particular.

4. The amount of gas in these XUV disks is enough to maintain the current level of star formation for several Gyr and, in some cases, up to a Hubble time. We cannot exclude the possibility that the $\mathrm{H}$ I associated with these disks may be the product of the dissociation of $\mathrm{H}_{2}$ in PDRs by the FUV radiation emitted by the stars formed during the current and recent episodes of XUV emission.

5. The observed colors and the level of chemical enrichment measured suggest that these XUV disks have experienced either (a) a continuous star formation for the last billion years or less or (b) an episodic star formation history with XUV episodes followed by quiescent periods. Should the latter scenario be correct, other (perhaps all) spiral galaxies might have gone through one or several of these episodes.

GALEX (Galaxy Evolution Explorer) is a NASA Small Explorer, launched in 2003 April. We gratefully acknowledge NASA's support for construction, operation, and science analysis for the GALEX mission, developed in cooperation with the Centre National d'Etudes Spatiales of France and the Korean Ministry of Science and Technology. We thank the anonymous referee for his/her constructive comments that have considerably improved the content of the paper. A. G. d. P. is financed by the MAGPOP EU Marie Curie Research Training Network and partially by the Spanish Programa Nacional de Astronomía y Astrofísica under grant AYA2003-01676. We thank Judith Cohen for kindly providing her $\mathrm{H} \alpha$ filter for COSMIC. We are also thankful to Sergio González and Wojtek Krzeminski for carrying out the imaging observations at the Las Campanas 40 inch telescope.

Facilities: GALEX, Magellan:Baade (IMACS), Hale (COSMIC), Swope (Direct CCD)

\section{REFERENCES}

Allen, R. J. 2002, in ASP Conf. Ser. 276, Seeing through the Dust: The Detection of $\mathrm{H}$ I and the Exploration of the ISM in Galaxies, ed. A. R. Taylor, T. L. Landecker, \& A. G. Willis (San Francisco: ASP), 288

Allende Prieto, C., Lambert, D. L., \& Asplund, M. 2001, ApJ, 556, L63

Avni, Y. 1976, ApJ, 210, 642

Baldwin, J. A., Phillips, M. M., \& Terlevich, R. 1981, PASP, 93, 5

Bianchi, L., et al. 2003, in The Local Group as an Astrophysical Laboratory, ed. M. Livio \& T. M. Brown (Baltimore: STScI), 10

Boissier, S., \& Prantzos, N. 1999, MNRAS, 307, 857

2000, MNRAS, 312, 398

Boissier, S., Prantzos, N., Boselli, A., \& Gavazzi, G. 2003, MNRAS, 346, 1215

Boissier, S., et al. 2007, ApJS, in press (astro-ph/0609071)

Brook, C. B., Kawata, D., Martel, H., Gibson, B. K., \& Bailin, J. 2006, ApJ, 639, 126

Bruzual, G., \& Charlot, S. 2003, MNRAS, 344, 1000

Bush, S. J., \& Wilcots, E. M. 2004, AJ, 128, 2789

Cardelli, J. A., Clayton, G. C., \& Mathis, J. S. 1989, ApJ, 345, 245

Cerviño, M. 1998, Ph.D. thesis, Univ. Complutense de Madrid

Clarke, C. J. 1989, MNRAS, 238, 283

Draine, B. T., \& Lee, H. M. 1984, ApJ, 285, 89

Elmegreen, B. G., \& Hunter, D. A. 2006, ApJ, 636, 712

Erwin, P., Beckman, J. E., \& Pohlen, M. 2005, ApJ, 626, L81

Ferguson, A. M. N. 1997, Ph.D. thesis, Johns Hopkins Univ.

Ferguson, A. M. N., Gallagher, J. S., \& Wyse, R. F. G. 1998, AJ, 116, 673

Ferland, G. J., Korista, K. T., Verner, D. A., Ferguson, J. W., Kingdon, J. B., \& Verner, E. M. 1998, PASP, 110, 761

Gallego, J., Zamorano, J., Rego, M., Alonso, O., \& Vitores, A. G. 1996, A\&AS, 120, 323

Galliano, F., Madden, S. C., Jones, A. P., Wilson, C. D., \& Bernard, J.-P. 2005, A\&A, 434, 867

Galliano, F., Madden, S. C., Jones, A. P., Wilson, C. D., Bernard, J.-P., \& Le Peintre, F. 2003, A\&A, 407, 159

Garnett, D. R., Shields, G. A., Skillman, E. D., Sagan, S. P., \& Dufour, R. J. 1997, ApJ, 489, 63

Gil de Paz, A., Madore, B. F., \& Pevunova, O. 2003, ApJS, 147, 29

Gil de Paz, A., et al. 2005, ApJ, 627, L29 2007, ApJS, in press (astro-ph/0606440)

Hillier, D. J. 2003, in IAU Symp. 212, A Massive Star Odyssey: From Main Sequence to Supernova, ed. K. van der Hucht, A. Herrero, \& C. Esteban (San Francisco: ASP), 70

Hollenbach, D., \& McKee, C. F. 1979, ApJS, 41, 555

Hollenbach, D., \& Salpeter, E. E. 1971, ApJ, 163, 155

Hoopes, C. G., et al. 2005, ApJ, 619, L99

Hou, J. L., Prantzos, N., \& Boissier, S. 2000, A\&A, 362, 921

Hughes, M. P., Thompson, A. R., \& Colvin, R. S. 1971, ApJS, 23, 323

Jones, T. W., \& Merrill, K. M. 1976, ApJ, 209, 509

Kehrig, C., Vílchez, J. M., Telles, E., Cuisinier, F., \& Pérez-Montero, E. 2006, A\&A, 457, 477

Kells, W., et al. 1998, PASP, 110, 1487

Kennicutt, R. C., Jr. 1989, ApJ, 344, 685

Kewley, L. J., \& Dopita, M. A. 2002, ApJS, 142, 35

Knapp, G. R., \& Kerr, F. J. 1974, A\&A, 35, 361

Kobulnicky, H. A., Kennicutt, R. C., Jr., \& Pizagno, J. L. 1999, ApJ, 514, 544

Kohta, N., Naomasa, N., \& Kuno, N. 2001, PASJ, 53, 757

Köppen, J., \& Hensler, G. 2005, A\&A, 434, 531

Kroupa, P., Tout, C. A., \& Gilmore, G. 1993, MNRAS, 262, 545

Larson, R. B. 1976, MNRAS, 176, 31

Leitherer, C., et al. 1999, ApJS, 123, 3

Lejeune, T., Cuisinier, F., \& Buser, R. 1997, A\&AS, 125, 229 
Liang, Y. C., Yin, S. Y., Hammer, F., Deng, L. C., Flores, H., \& Zhang, B. 2006, ApJ, 652, 257

Madore, B. F., et al. 2004, BAAS, 36, 1554

Martin, C. L., \& Kennicutt, R. C., Jr. 2001, ApJ, 555, 301

Martin, D. C., et al. 2005, ApJ, 619, L1

Matteucci, F., \& Francois, P. 1989, MNRAS, 239, 885

McGaugh, S. S. 1991, ApJ, 380, 140 (M91)

Meurer, G. R., et al. 2004, BAAS, 36, 1410

Mihos, J. C., Spaans, M., \& McGaugh, S. S. 1999, ApJ, 515, 89

Mollá, M., Vílchez, J. M., Gavilán, M., \& Díaz, A. I. 2006, MNRAS, 372, 1069

Muñoz-Mateos, J. C., Gil de Paz, A., Boissier, S., Zamorano, J., Jarrett, T., Gallego, J., \& Madore, B. F. 2007, ApJ, 658, 1006

Osterbrock, D. E. 1989, Astrophysics of Gaseous Nebulae and Active Galactic Nuclei (Mill Valley: University Science Books)

Pagel, B. E. J., Edmunds, M. G., Blackwell, D. E., Chun, M. S., \& Smith, G. 1979, MNRAS, 189, 95

Pauldrach, A. W. A. 2005, in AIP Conf. Proc. 804, Planetary Nebulae as Astronomical Tools, ed. R. Szczerba, G. Stasinska, \& S. K. Górny (Melville: AIP), 105

Pilyugin, L. S. 2000, A\&A, 362, 325

Pilyugin, L. S., \& Thuan, T. X. 2005, ApJ, 631, 231 (PT05)

Pilyugin, L. S., Thuan, T. X., \& Vílchez, J. M. 2003, A\&A, 397, 487

Popescu, C. C., \& Tuffs, R. J. 2003, A\&A, 410, L21

Prantzos, N., \& Boissier, S. 2000, MNRAS, 313, 338

Prochaska, J. X., et al. 2002, PASP, 114, 933

Salzer, J. J., Lee, J. C., Melbourne, J., Hinz, J. L., Alonso-Herrero, A., \& Jangren, A. 2005, ApJ, 624, 661
Schaerer, D., \& de Koter, A. 1997, A\&A, 322, 598

Schlegel, D. J., Finkbeiner, D. P., \& Davis, M. 1998, ApJ, 500, 525

Smith, L. J., Norris, R. P. F., \& Crowther, P. A. 2002, MNRAS, 337, 1309

Spitzer, L. 1978, Physical Processes in the Interstellar Medium (New York: Wiley)

Stasinska, G., \& Leitherer, C. 1996, ApJS, 107, 661

Stasinska, G., \& Schaerer, D. 1997, A\&A, 322, 615

Sternberg, A., Hoffmann, T. L., \& Pauldrach, A. W. A. 2003, ApJ, 599, 1333

Swaters, R. A., \& Balcells, M. 2002, A\&A, 390, 863

Thilker, D. A., et al. 2005a, ApJ, 619, L79 2005b, BAAS, 37, 1500

Tilanus, R. P. J., \& Allen, R. J. 1993, A\&A, 274, 707

Tout, C. A., Pols, O. R., Eggleton, P. P., \& Han, Z. 1996, MNRAS, 281, 257

van Zee, L., \& Haynes, M. P. 2006, ApJ, 636, 214

van Zee, L., Salzer, J. J., \& Haynes, M. P. 1998a, ApJ, 497, L1

van Zee, L., Salzer, J. J., Haynes, M. P., O’Donoghue, A. A., \& Balonek, T. J. 1998b, AJ, 116, 2805

Veilleux, S., \& Osterbrock, D. E. 1987, ApJS, 63, 295

Vidali, G., Roser, J., Manicó, G., Pirronello, V., Perets, H. B., \& Biham, O. 2005, J. Phys. Conf. Ser., 6, 36

Webster, B. L., \& Smith, M. G. 1983, MNRAS, 204, 743

Zamorano, J., Gallego, J., Rego, M., Vitores, A. G., \& Alonso, O. 1996, ApJS, 105,343

Zamorano, J., Rego, M., Gallego, J., Vitores, A. G., González-Riestra, R., \& Rodríguez-Caderot, G. 1994, ApJS, 95, 387 